\title{
Limit cycles and conformal invariance
}

\author{
Jean-François Fortin, ${ }^{a, b, c}$ Benjamín Grinstein $^{c}$ and Andreas Stergiou ${ }^{c}$ \\ a Theory Division, Department of Physics, CERN, \\ CH-1211 Geneva 23, Switzerland \\ ${ }^{b}$ Stanford Institute for Theoretical Physics, Department of Physics, Stanford University, \\ Stanford, CA 94305, U.S.A. \\ ${ }^{c}$ Department of Physics, University of California, \\ San Diego, La Jolla, CA 92093 U.S.A. \\ E-mail: jean-francois.fortin@cern.ch, bgrinstein@ucsd.edu, \\ stergiou@physics.ucsd.edu
}

ABSTRACT: There is a widely held belief that conformal field theories (CFTs) require zero beta functions. Nevertheless, the work of Jack and Osborn implies that the beta functions are not actually the quantites that decide conformality, but until recently no such behavior had been exhibited. Our recent work has led to the discovery of CFTs with nonzero beta functions, more precisely CFTs that live on recurrent trajectories, e.g., limit cycles, of the beta-function vector field. To demonstrate this we study the $S$ function of Jack and Osborn. We use Weyl consistency conditions to show that it vanishes at fixed points and agrees with the generator $Q$ of limit cycles on them. Moreover, we compute $S$ to third order in perturbation theory, and explicitly verify that it agrees with our previous determinations of $Q$. A byproduct of our analysis is that, in perturbation theory, unitarity and scale invariance imply conformal invariance in four-dimensional quantum field theories. Finally, we study some properties of these new, "cyclic" CFTs, and point out that the $a$-theorem still governs the asymptotic behavior of renormalization-group flows.

Keywords: Conformal and W Symmetry, Space-Time Symmetries, Renormalization Group

ArXiv ePRINT: 1208.3674 


\section{Contents}

1 Overview in lieu of introduction 1

2 Weyl consistency conditions $\quad 5$

2.1 The trace anomaly and the computation of $\nabla_{\mu} \mathscr{Z}^{\mu} \quad 10$

2.2 Wess-Zumino action 11

3 Flavor symmetries, dimension-three operators and the corrected trace anomaly

4 Scale implies conformal invariance $\quad 17$

$\begin{array}{lll}4.1 & S \text { is } Q \text { (on cycles) } & 17\end{array}$

4.2 Cyclic CFTs 19

4.2.1 A perturbative proof that scale imples conformal invariance $\quad 19$

$\begin{array}{ll}\text { 4.2.2 Some properties of cyclic CFTs } & 19\end{array}$

5 The $c$-theorem in the presence of cycles 20

$6 \quad$ Summary and concluding remarks $\quad 23$

$\begin{array}{ll}\text { A Ambiguities in RG functions } & 24\end{array}$

B The relation between the metrics $\chi_{i j}^{a}$ and $\chi_{i j}^{g} \quad \mathbf{2 5}$

$\begin{array}{ll}\text { C How to calculate } N_{I} \text { and } S & \mathbf{2 7}\end{array}$

$\begin{array}{ll}\text { C.1 One loop } & 27\end{array}$

$\begin{array}{lll}\text { C.2 Two loops } & 28\end{array}$

$\begin{array}{lll}\text { C.3 Three loops } & 29\end{array}$

\section{Overview in lieu of introduction}

Two recent reported results can potentially greatly enrich our understanding of quantum field theory (QFT). On the one hand, Komargodski and Schwimmer (KS) [1], following earlier work by Cappelli, D'Appollonio, Guida and Magnoli (CDGM) [2, 3], have delineated a nonperturbative proof of an inequality satisfied when a four-dimensional QFT flows between two fixed points of the renormalization group (RG). On the other hand, we have discovered closed RG trajectories ${ }^{1}$ in theories in $d=4-\epsilon[4-6]$ and $d=4$ [7] spacetime

\footnotetext{
${ }^{1}$ Meaning closed flow-lines of the familiar dim-reg beta-function vector field, in conventions where the anomalous-dimension matrix is symmetric. For a word on conventions and their effects on RG functions see appendix A.
} 
dimensions, in a regime where perturbation theory is applicable. While the former result can impose restrictions on the possible realizations of long distance (IR) phases of QFTs, the latter exhibits explicitly a novel feature of QFTs. A question naturally arises as to whether these results are compatible.

In this work we will show perturbatively that unitary, interacting, scale-invariant cycles $^{2}$ in $d=4$ correspond to conformal field theories (CFTs), that is, theories with invariance under the full conformal group, not just Poincaré plus dilatations. This follows from the work of Jack and Osborn (JO) [8]. Compatibility of this type of cycles ${ }^{3}$ with the aforementioned inequality is then not surprising since the inequality still compares a quantity defined on CFTs, be it a CFT at an endpoint of an RG flow or a CFT corresponding to a limit cycle of the RG flow.

Luty, Polchinski and Rattazzi (LPR) [11] argued that limit cycles cannot exist in $d=4$ unitary QFT, and hence that scale without conformal invariance is excluded. As we shall see, limit cycles do occur, but QFTs on them are fully conformal, not just scale-invariant. LPR have informed us that their manuscript is being replaced with one that contains a corrected version of their argument, with their conclusion regarding the absence of scale without conformal invariance unchanged.

The work of KS is not sensitive to the presence of cycles. Indeed, KS assume the existence of a flow from a short distance (UV) CFT to an IR CFT, and argue that the coefficient $a$ of the Euler density in the curved-space trace anomaly,

$$
T_{\mu}^{\mu}=\text { operator terms }+c(\text { Weyl tensor squared })-a(\text { Euler density })
$$

is larger at the UV than the IR fixed point: $a_{\mathrm{UV}}>a_{\mathrm{IR}}$. This, then, is a proof of the "weak version" of the $c$-theorem. The KS argument incorporates putative flows from a fixed point or cycle to another fixed point or cycle, since in both cases the theories encountered are CFTs.

In $d=2$ a stronger result holds: there exists a quantity $c$, local in the RG scale, that is monotonically decreasing along any RG flow [12]. This is referred to as the "strong version" of the $c$-theorem, and it was first argued to also be true in $d=4$ by Cardy [13]. A proof was later found by JO (see also [14]), albeit only in perturbation theory. Away from fixed points the quantity that plays the role of $c$ in the arguments of JO is not exactly equal to $a$ (the coefficient of the Euler density in the curved-space trace anomaly). However, it agrees with $a$ at endpoints/limit cycles of the RG trajectories. This is in agreement with the result of KS that the weak version of the $c$-theorem is valid for $a$. In this paper we extend the perturbative proof of JO to include RG cycles.

Of course it is well-known that a may increase away from trivial UV fixed points: for example, for pure Yang-Mills (YM) theory with beta function $\beta^{g}=-\beta_{0} g^{3} / 16 \pi^{2}-$

\footnotetext{
${ }^{2}$ More precisely "limit recursive flows" of the dim-reg beta-function vector field. In what follows we refer to both limit cycles and limiting ergodic behavior simply as "cycles."

${ }^{3}$ The condition for scale invariance, $\mu d g^{i} / d \mu=Q_{j}^{i} g^{j}, Q^{T}=-Q=$ constant [9], gives recursive flows [10]. Our study of cycles here is concerned with this type of closed trajectories, given by a rotation of the coupling constants by a compact Abelian group generated by $Q$. Whether recursive flows that are not of this type exist is an open question.
} 
$\beta_{1} g^{5} /\left(16 \pi^{2}\right)^{2}-\cdots$ one has $[8]$

$$
a=a_{0}+\frac{n_{V} \beta_{1}}{8\left(16 \pi^{2}\right)^{3}} g^{4}+\mathcal{O}\left(g^{6}\right) .
$$

Here $a_{0}$ is the free field theory (one-loop) value of $a$ and $n_{V}=\operatorname{dim}(\operatorname{Adj})$ is the number of vector fields. ${ }^{4}$ Nevertheless, even in this case JO showed that there exists a quantity, $\tilde{\beta}_{b}$, which flows monotonically (to all orders in perturbation theory). The quantity $\tilde{\beta}_{b}$ is related to $a$, which in $\mathrm{JO}$ is denoted by $\beta_{b}$, by

$$
\tilde{\beta}_{b}=\beta_{b}+\frac{1}{8} w \beta^{g}, \quad \beta_{b} \equiv a .
$$

Here $w$ is a function of the coupling $g$, and $\beta^{g}=-d g / d t$ is the beta function. While $\tilde{\beta}_{b}$ and $\beta_{b}$ agree on fixed points, the difference is parametrically large away from fixed points. In section 2 we explain this in detail.

The result of JO follows from careful inspection of how the theory responds to Weyl rescaling. The KS method, or an elaboration on it by LPR [11], extensively uses Weyl rescaling and takes advantage of the particularly simple form this takes on fixed points. However, in trying to extend the KS arguments to produce a proof of the strong version of the $c$-theorem, LPR use Weyl rescaling away from fixed points. We explain how consistency requires introducing spacetime-dependent coupling constants and then in addition new counterterms that involve derivatives of the couplings. We use the very rescaling in LPR to derive JO's consistency conditions anew, of which the monotonic flow of $\tilde{\beta}_{b}$ is but one example.

For models which display cycles the state of affairs is significantly more complex. In all these models the kinetic terms of the Lagrangian are invariant under a "flavor" symmetry group $G_{F}$ (that commutes with the gauge group). Scalar self-interactions and Yukawa couplings of scalars with fermions break $G_{F}$. The dependence of counterterms on the coupling constants characterizing these interactions is restricted by the pattern of breaking of $G_{F}$. There is a well-known, simple method of accounting for this. The coupling constants are treated as spurions, that is, as non-dynamical fields, and allowed to transform under $G_{F}$ precisely so as to render the Lagrangian invariant under these symmetry transformations. Then, if the regulator respects the symmetry, so will the counterterms. It follows that the entries in the trace anomaly respect the symmetry too. As $a$ is the coefficient of the $G_{F}$-invariant Euler density, it is itself $G_{F}$-invariant as well. And since the flow on a cycle corresponds to a $G_{F}$-transformation of the couplings, $a$ remains constant on the cycle.

This raises the following question: "how is the monotonic flow of $\tilde{\beta}_{b}$ consistent with the constancy of $a$ ?" Actually, $\tilde{\beta}_{b}$ is also $G_{F}$-invariant, and is thus also guaranteed to be constant along the cyclic flow. The answer is found in the flow equation for $\tilde{\beta}_{b}$ given by Osborn in [14]. His equation is a generalization, applicable to these more complex theories, of that found by JO. This flow equation is not guaranteed to give monotonic flows, but can and does give constancy of $\tilde{\beta}_{b}$ on cycles. We review the work of JO concerning these more complex theories in section 3 , and show that a quantity $\widetilde{B}_{b}$ decreases monotonically along

\footnotetext{
${ }^{4}$ We thank K. Intriligator for discussions on this point.
} 
RG flows, at least in perturbation theory, and agrees with $\beta_{b}$ on fixed points and cycles. This is a result essentially contained in $[8,14]$, although it is not explicitly mentioned there.

To obtain this result an understanding of the modifications to the trace-anomaly equation in theories with cycles is required. It is a little-known fact that in theories with many fermions and scalars there generically appears a term in the trace anomaly of the form of the divergence of a current. The current generates transformations that correspond to a particular element $S$ of the Lie algebra of $G_{F}$, that is a function of the coupling constants. JO showed by direct computation that $S$ vanishes to three loops if the field content of the QFT consists solely of scalars, and to two loops if of scalars and Dirac spinors. On the other hand, the element $Q$ of the Lie algebra of $G_{F}$ that generates the flow along the cycle is found in our computations to arise at three-loop order in gauge theories that include both scalars and spinors [7]. Could it be, then, that $S$ is non-zero at three loops in these theories? And if so, what is the relation between $Q$ and $S$ ? In section 4.1 we take on the task of computing the lowest-order contribution to JO's $S$ for the most general fourdimensional QFT, compare with $Q$ and demonstrate that $S$ agrees with $Q$ on cycles and vanishes on fixed points. A corollary of this result is that scale implies conformal invariance in relativistic unitary perturbative four-dimensional QFTs.

That $S$ agrees with $Q$ on cycles suggests that the two terms in the flat-space trace anomaly may cancel. That is, the well-known $\beta \partial \mathscr{L} / \partial g$ term may cancel against the littleknown divergence of the $S$-current term, since the $\beta$-term is determined by $Q$ on cycles. This is indeed what happens: the trace of the stress-energy tensor vanishes for unitary, scale-invariant cycles, and hence these models display invariance under the full conformal group. In the rest of section 4.2 we prove this and explore a few of its consequences. Armed with these results, we return to the proof of the $c$-theorem in section 5 . There we give a slightly streamlined version of the LPR version of the KS argument, with care to address the possible differences that may arise when the CFTs at the ends of the RG flow correspond to cycles.

Let us specify here that we follow closely the notation of JO [8], with some notable exceptions. From here on, following JO, in the anomaly equation we use $\beta_{a}$ and $\beta_{b}$ for $-c$ and $a$ respectively, although we still use the terminology $c$-theorem instead of the more accurate $\beta_{b}$-theorem. Also, we call $\lambda_{a, b, c}$ rather than $a, b, c$ the infinite counterterms that give rise to $\beta_{a, b, c}$ (having infinite counterterms labeled by $a$ and $c$ can certainly produce confusion with the corresponding "beta functions" that appear in the Weyl anomaly and that are commonly referred to as $a$ and $c$ ). Throughout this paper RG time is defined by $t=-\ln \left(\mu / \mu_{0}\right)$, so that it increases as we flow to the IR.

Many of our results are extracted from the work of JO. So it is perhaps necessary to remark that, besides parsing the results of JO to hopefully make them slightly more accessible to the general reader, we have made several novel contributions:

- We have discovered where in the argument of LPR the quantity $\beta_{b}$ is replaced by $\tilde{\beta}_{b}$ (or, in more generality, by $\widetilde{B}_{b}$ ).

- We have extended JO's calculation of $S$ to third loop order, which is the leading non-vanishing contribution to $S$ in a Yang-Mills theory with scalars and spinors. 
- We have shown that $S$ vanishes on fixed points and agrees with the generator $Q$ of limit cycles on them.

- We have demonstrated in perturbation theory that unitary, scale and Poincaré invariant, interacting QFTs in $d=4$ have vanishing trace of the stress-energy tensor and hence are invariant under the full conformal group.

- We have used the above to

o find, using arguments of JO, a perturbative proof of an extension of the strong version of the $c$-theorem, i.e., that there exists a quantity that monotonically decreases in flows out of UV fixed points and cycles, and

- clarify that the arguments of KS apply even in the presence of cycles, i.e., that $\left(\beta_{b}\right)_{\mathrm{UV}}>\left(\beta_{b}\right)_{\mathrm{IR}}$ for presumed RG flows that can now originate or terminate on cycles as well as fixed points, valid even outside perturbation theory (provided the implicit assumptions in KS do not invalidate their result).

\section{Weyl consistency conditions}

In this section we review the derivation of the Weyl consistency conditions of JO. The method uses as a starting point the expressions of Weyl invariance used by KS and by LPR. The presentation is formulated so that it becomes clear that the assumptions in those works already implicitly lead to the JO consistency conditions. Hence, although the derivation presented here may seem novel, it actually follows closely JO. We have included it here for completeness, for pedagogy and because it makes clear that neither the results of KS nor those of LPR should be in conflict with those of JO.

Let us briefly review Osborn's argument for the consistency conditions [14]. These are analogous to the well-known Wess-Zumino consistency conditions [15]. Let $\Delta_{\sigma} \widetilde{W}$ denote the action of a Weyl transformation on $\widetilde{W}$, the generating functional for connected renormalized Green functions. Because of the Abelian nature of the Weyl group, the Weyl consistency conditions follow:

$$
\left[\Delta_{\sigma}, \Delta_{\sigma^{\prime}}\right] \widetilde{W}=0
$$

In JO the same consistency conditions are obtained by requiring finiteness of the trace of the stress-energy tensor in curved background and with spacetime-dependent couplings. One can also obtain the Weyl consistency conditions based on the arguments of LPR.

LPR start from a quantum action $S_{0}$ which is a function of a conformally flat metric, $\gamma_{\mu \nu}=e^{-2 \tau(x)} \eta_{\mu \nu}$ and coupling constants $g^{i}(\mu)$ (in $d=4-\epsilon$ regularization, with, say, minimal subtraction (MS)). By rescaling the fields, which are dummy variables of integration anyway, by $\phi \rightarrow\left(e^{\tau}\right)^{\delta} \phi$, where $\delta$ is the canonical dimension of the field (as in $\delta=(d-2) / 2$ for scalars $)$, and using the $\mu$-independence of the bare couplings, one sees that the $\tau$-dependence in $S_{0}$ arises only due to the scale dependence of renormalized coupling constants, $g^{i}\left(e^{\tau} \mu\right)$. Effectively, the regularized generating functional $W$ satisfies

$$
W\left[e^{-2 \tau(x)} \eta_{\mu \nu}, g^{i}(\mu)\right]=W\left[\eta_{\mu \nu}, g^{i}\left(e^{\tau(x)} \mu\right)\right] .
$$


Alternatively, Komargodski [16] argues that the functional is made invariant under Weyl transformations by adding a conformal compensator $\tau(x)$. One can write

$$
W\left[e^{-2 \tau(x)} \eta_{\mu \nu}, g^{i}\left(e^{-\tau(x)} \mu\right)\right]=W\left[\eta_{\mu \nu}, g^{i}(\mu)\right],
$$

or, equivalently, that the left-hand side is invariant under $\tau \rightarrow \tau+\sigma$. For finiteness it is also necessary to include in $W$ all possible counterterms that are functions of spacetimedependent background and coupling constants, $\gamma_{\mu \nu}(x)$ and $g^{i}(x)$. It is from counterterms that do not vanish for spacetime-independent coupling constants that the $\beta_{a, b, c}$-anomalies arise. It is convenient, in order to keep track of curvature-dependent terms, to do this in a more general background metric,

$$
\begin{aligned}
W\left[e^{-2 \tau(x)} \gamma_{\mu \nu}(x), g^{i}(\mu)\right] & =W\left[\gamma_{\mu \nu}(x), g^{i}\left(e^{\tau(x)} \mu\right)\right], \\
W\left[e^{-2 \tau(x)} \gamma_{\mu \nu}(x), g^{i}\left(e^{-\tau(x)} \mu\right)\right] & =W\left[\gamma_{\mu \nu}(x), g^{i}(\mu)\right] .
\end{aligned}
$$

At the risk of restating the trivial, let us emphasize that it is not consistent to neglect the spacetime dependence of couplings when studying Weyl anomalies, since the Weyl transformation involves spacetime-dependent couplings. The counterterms associated with spacetime derivatives of these couplings will lead to additional anomalies. Some of these may - and as we will see, do - contribute to the dilaton/compensator scattering amplitude even after one takes the limit of flat background and spacetime-independent coupling constants.

The approach of LPR allows one to compute quantities associated with a model in a curved background with spacetime-independent coupling constants in terms of corresponding quantities for the same model but in a flat background with, however, spacetimedependent coupling constants. This observation is not new. For example, in JO the same observation is used precisely for the same purpose, namely, to compute the anomalies associated with scale transformations using only computations in flat space. Similarly, the approach of Komargodski allows for an explicit nonlinear realization of scale invariance, at the price of introducing spacetime-dependent coupling constants. In either case it is important to realize that new counterterms are required to render the model finite, much like counterterms involving derivatives of the metric need to be introduced to render finite the model in a curved background. These new counterterms must involve derivatives of the coupling constants and lead to new Weyl anomalies. At the end of this section we study how these new anomalies contribute to the Wess-Zumino action for the conformal compensator $\tau(x)$ even after the couplings and the metric are taken to be spacetime-independent. For the remainder of this section we take a closer look at these counterterms, the anomalies they produce and the relations between them, that is, the JO consistency conditions, that follow from (2.2).

Consider the theory in the background of an arbitrary metric $\gamma_{\mu \nu}(x)$ and arbitrary spacetime-dependent coupling constants $g^{i}(x)$ corresponding to interaction terms $g^{i}(x) \mathcal{O}_{i}(x)$ in the Lagrangian. The arbitrary spacetime dependence of the couplings allows one to use them as sources for operators in the interaction part of the Lagrangian, by taking functional derivatives of the generating functional with respect to $g^{i}(x)$. If the 
quantum action is renormalized, then this procedure automatically gives finite correlations functions for the insertions of these operators. Let $\widetilde{W}$ stand for the renormalized generating functional. It is convenient to separate the counterterms that are independent of quantum fields from the rest of the action. They can be taken out of the functional integral and contribute directly to $\widetilde{W}$ :

$$
\widetilde{W}=W+W_{\text {c.t. }}
$$

The generating functional $W$ results from performing the functional integral over quantum fields in the absence of the quantum-field-independent counterterms. The counterterms required to render the theory finite were first classified in JO. They consist of all possible diff-invariant dimension-four operators constructed out of the metric and couplings and their derivatives:

$$
W_{\text {c.t. }}=-\int \sqrt{-\gamma} \mu^{-\epsilon} \lambda \cdot \mathscr{R},
$$

where dimensional regularization is used with $d=4-\epsilon$ and

$$
\begin{aligned}
\lambda \cdot \mathscr{R}= & \lambda_{a} F+\lambda_{b} G+\lambda_{c} H^{2}+\mathscr{E}_{i} \partial_{\mu} g^{i} \partial^{\mu} H+\frac{1}{2} \mathscr{F}_{i j} \partial_{\mu} g^{i} \partial^{\mu} g^{j} H+\frac{1}{2} \mathscr{G}_{i j} \partial_{\mu} g^{i} \partial_{\nu} g^{j} G^{\mu \nu} \\
& +\frac{1}{2} \mathscr{A}_{i j} \nabla^{2} g^{i} \nabla^{2} g^{j}+\frac{1}{2} \mathscr{B}_{i j k} \partial_{\mu} g^{i} \partial^{\mu} g^{j} \nabla^{2} g^{k}+\frac{1}{4} \mathscr{C}_{i j k l} \partial_{\mu} g^{i} \partial^{\mu} g^{j} \partial_{\nu} g^{k} \partial^{\nu} g^{l}
\end{aligned}
$$

Here $F$ is the Weyl tensor squared, $G$ is the Euler density, $H$ is proportional to the Ricci scalar, and $G_{\mu \nu}$ is the Einstein tensor:

$$
\begin{aligned}
& F=R^{\mu \nu \rho \sigma} R_{\mu \nu \rho \sigma}-\frac{4}{d-2} R^{\mu \nu} R_{\mu \nu}+\frac{2}{(d-2)(d-1)} R^{2}, \\
& G=\frac{2}{(d-3)(d-2)}\left(R^{\mu \nu \rho \sigma} R_{\mu \nu \rho \sigma}-4 R^{\mu \nu} R_{\mu \nu}+R^{2}\right), \\
& H=\frac{1}{d-1} R, \quad G_{\mu \nu}=\frac{2}{d-2}\left(R_{\mu \nu}-\frac{1}{2} \gamma_{\mu \nu} R\right) .
\end{aligned}
$$

The quantities above are defined as in JO, with inessential $d$-dependent factors for later convenience. Each of the counterterms in $\lambda \cdot \mathscr{R}$ is an expansion in $1 / \epsilon$ chosen to render $\widetilde{W}$ finite - for this one must in addition introduce wave-function and coupling constant counterterms, as usual. The coefficients $\lambda=\left(\lambda_{a}, \lambda_{b}, \ldots, \mathscr{C}_{i j k l}\right)$ are in general functions of the couplings $g^{i}(x)$.

The anomalous variation of the generating functional is dictated by these counterterms. While $W$ satisfies (2.1) and (2.2), this is not true of $W_{\text {c.t. }}$, as can be seen by explicit computation. The anomaly is precisely the statement that the infinitesimal transformation $\tau \rightarrow \tau+\sigma$ in $(2.2 \mathrm{~b})$

$$
\Delta_{\sigma} W_{\text {c.t. }}=W_{\text {c.t. }}\left[(1-2 \sigma) \gamma_{\mu \nu}, g^{i}-\sigma \mu d g^{i} / d \mu\right]-W_{\text {c.t. }}\left[\gamma_{\mu \nu}, g^{i}\right],
$$

fails to vanish. The anomalous variation can be split into a term that would occur even if $\sigma$ were spacetime-independent plus a term proportional to the derivative of $\sigma$ :

$$
\Delta W_{\text {anomaly }}=\Delta_{\sigma} W_{\text {c.t. }}=-\int \sqrt{-\gamma}\left(\sigma \beta_{\lambda} \cdot \mathscr{R}+\partial_{\mu} \sigma \mathscr{Z}^{\mu}\right) .
$$


These terms again can be expanded using dimensional analysis and diff-invariance:

$$
\begin{aligned}
\beta_{\lambda} \cdot \mathscr{R}= & \beta_{a} F+\beta_{b} G+\beta_{c} H^{2}+\chi_{i}^{e} \partial_{\mu} g^{i} \partial^{\mu} H+\frac{1}{2} \chi_{i j}^{f} \partial_{\mu} g^{i} \partial^{\mu} g^{j} H+\frac{1}{2} \chi_{i j}^{g} \partial_{\mu} g^{i} \partial_{\nu} g^{j} G^{\mu \nu} \\
& +\frac{1}{2} \chi_{i j}^{a} \nabla^{2} g^{i} \nabla^{2} g^{j}+\frac{1}{2} \chi_{i j k}^{b} \partial_{\mu} g^{i} \partial^{\mu} g^{j} \nabla^{2} g^{k}+\frac{1}{4} \chi_{i j k l}^{c} \partial_{\mu} g^{i} \partial^{\mu} g^{j} \partial_{\nu} g^{k} \partial^{\nu} g^{l}
\end{aligned}
$$

and $^{5}$

$$
\begin{aligned}
\mathscr{Z}_{\mu}= & G_{\mu \nu} w_{i} \partial^{\nu} g^{i}+\partial_{\mu}(H d)+H Y_{i} \partial_{\mu} g^{i} \\
& +\partial_{\mu}\left(U_{i} \nabla^{2} g^{i}+\frac{1}{2} V_{i j} \partial_{\nu} g^{i} \partial^{\nu} g^{j}\right)+S_{i j} \partial_{\mu} g^{i} \nabla^{2} g^{j}+\frac{1}{2} T_{i j k} \partial_{\nu} g^{i} \partial^{\nu} g^{j} \partial_{\mu} g^{k},
\end{aligned}
$$

up to terms with vanishing divergence. Since $\widetilde{W}$ is finite and the $\sigma$-variation of $W$ vanishes, it must be that the variation of $W_{\text {c.t. }}$ is finite by itself.

Calculations of the coefficients in $\beta_{\lambda} \cdot \mathscr{R}$ and $\mathscr{Z}_{\mu}$ can be done using standard techniques of dimensional regularization with a mass-independent renormalization scheme, say MS. For now, let us concentrate on the relatively straightforward computation of $\beta_{\lambda} \cdot \mathscr{R}$. Since for constant $\sigma$ the transformation $\delta \gamma_{\mu \nu}=-2 \sigma \gamma_{\mu \nu}$ just counts dimensions, and the dimension of the volume element is $d$ while that of the operators in $W_{\text {c.t. }}$ is four, we obtain

$$
\left(\epsilon-\hat{\beta}^{i} \hat{\partial}_{i}\right) \lambda \cdot \mathscr{R}=\beta_{\lambda} \cdot \mathscr{R},
$$

where the beta function is

$$
\mu \frac{d g^{i}}{d \mu}=\hat{\beta}^{i}=-\epsilon k^{i} g^{i}+\beta^{i}(g) \quad(\text { no sum over } i) .
$$

Here the derivative is taken holding the bare parameters fixed. $k^{i}$ is defined by requiring that the Lagrangian scales appropriately: for $\phi^{\prime}=\mu^{\delta \epsilon} \phi$ and $g^{\prime i}=\mu^{k^{i} \epsilon} g^{i}$, then $\mathscr{L}\left(\phi^{\prime}, g^{\prime}\right)=$ $\mu^{-\epsilon} \mathscr{L}(\phi, g)$. Note that $\hat{\beta}^{i} \hat{\partial}_{i} \equiv \hat{\beta}^{i} \hat{\partial} / \hat{\partial} g^{i}$ denotes substitution of $g^{i}$ by $\beta^{i}$ wherever $g^{i}$ may be found, e.g., $\hat{\beta}^{i} \hat{\partial}_{i}\left(\partial_{\mu} g^{j}\right) \equiv \partial_{\mu} \beta^{j}=\partial_{i} \beta^{j} \partial_{\mu} g^{i}$, and of course respects the standard rules of differentiation. Using (2.7), it is straightforward to show that, e.g.,

$$
\chi_{i j}^{a}=\left(\epsilon-\hat{\beta}^{k} \partial_{k}\right) \mathscr{A}_{i j}-\mathscr{A}_{i k} \partial_{j} \hat{\beta}^{k}-\mathscr{A}_{j k} \partial_{i} \hat{\beta}^{k} .
$$

The consistency conditions of JO can be understood as following from requiring that (2.2) applied to the complete renormalized generating function $\widetilde{W}$ fails only up to the finite, anomaly terms. The left-hand side of (2.2a) does not involve any counterterms from spacetime-dependent couplings, while the right-hand side does not involve any from a curved background. Hence, the counterterms in one and the other case must be related. Consider on the right-hand side of (2.2a), for example, the counterterm

$$
\frac{1}{2} \mathscr{A}_{i j} \nabla^{2} g^{i} \nabla^{2} g^{j}=\frac{1}{2} \mathscr{A}_{i j} \hat{\beta}^{i} \hat{\beta}^{j}\left(\nabla^{2} \tau\right)^{2}+\cdots,
$$

\footnotetext{
${ }^{5}$ The second term involves the function of coupling constants $d$, which is not to be confused with $d=4-\epsilon$. We follow Osborn in this unfortunate choice of notation, hoping that with this warning no confusion will arise in what follows.
} 
where we have expanded to lowest order in $\tau(x)$. Comparing with the counterterms on the left-hand side of (2.2a), that arise solely from a curved background, we have,

$$
\sqrt{-\gamma}\left(\lambda_{a} F+\lambda_{b} G+\lambda_{c} H^{2}\right)=8 \lambda_{b}\left[\left(\nabla^{2} \tau\right)^{2}-\left(\partial_{\mu} \partial_{\nu} \tau\right)^{2}+\cdots\right]+4 \lambda_{c}\left[\left(\nabla^{2} \tau\right)^{2}+\cdots\right] .
$$

The $\lambda_{b}$ term is a total derivative so for localized $\tau(x)$ it does not contribute (recall there is an implicit spacetime integration). Matching the terms in (2.8) and (2.9) we find that the counterterms are related,

$$
4 \lambda_{c} \sim \frac{1}{2} \mathscr{A}_{i j} \hat{\beta}^{i} \hat{\beta}^{j},
$$

where the symbol $\sim$ denotes equality up to finite terms, that is, the difference is finite as $\epsilon \rightarrow 0$. This precisely corresponds to eq. (3.12) of JO. Applying $\mu d / d \mu$ on the bare couplings to derive RGEs and the corresponding beta functions, one then derives from this JO's consistency condition (3.21a),

$$
8 \beta_{c}=\chi_{i j}^{a} \beta^{i} \beta^{j}-\beta^{i} \partial_{i} X
$$

where $X$ arises from the finite difference between the left- and right-hand sides of (2.10), and $\beta_{c}$ and $\chi_{i j}^{a}$ are beta functions for $\lambda_{c}$ and $\mathscr{A}_{i j}$, respectively. The remaining consistency conditions in JO can be obtained in a similar fashion. We only quote here one other consistency condition that plays an important role in what follows. Using (2.2a) the lowest order terms in $\tau(x)$ that are linear in the Einstein tensor give

$$
8 \partial_{i} \lambda_{b} \sim \mathscr{G}_{i j} \hat{\beta}^{j}
$$

With the finite difference between the two sides of (2.12) denoted by $w_{i}$ one obtains

$$
8 \partial_{i} \beta_{b}=\chi_{i j}^{g} \beta^{j}-\beta^{j} \partial_{j} w_{i}-\partial_{i} \beta^{j} w_{j}
$$

This consistency condition is the origin of the proposal in JO for a $c$-function,

$$
\tilde{\beta}_{b} \equiv \beta_{b}+\frac{1}{8} \beta^{i} w_{i}
$$

which satisfies

$$
\partial_{i} \tilde{\beta}_{b}=\frac{1}{8}\left(\chi_{i j}^{g}+\partial_{[i} w_{j]}\right) \beta^{j},
$$

where $\partial_{[i} w_{j]}=\partial_{i} w_{j}-\partial_{j} w_{i}$. Then its RG flow is monotonic provided the "metric" $\chi_{i j}^{g}$ is positive-definite, for

$$
-\frac{d \tilde{\beta}_{b}}{d t}=\beta^{i} \partial_{i} \tilde{\beta}_{b}=\frac{1}{8} \chi_{i j}^{g} \beta^{i} \beta^{j} .
$$

To summarize, the extension (2.2) of the invariance requirement of LPR in (2.1), when applied to the complete set of counterterms required for finiteness when coupling constants have spacetime dependence, leads to the consistency conditions of JO. 


\subsection{The trace anomaly and the computation of $\nabla_{\mu} \mathscr{Z}^{\mu}$}

As formulated, the renormalized generating functional $\widetilde{W}$ is a finite function of the background metric and of renormalized spacetime-dependent coupling constants. As such we can obtain finite insertions of composite operators in Green functions by functional differentiation,

$$
\left\langle T_{\mu \nu}(x)\right\rangle=\frac{2}{\sqrt{-\gamma}} \frac{\delta \widetilde{W}}{\delta \gamma^{\mu \nu}(x)} \quad \text { and } \quad\left\langle\left[\mathcal{O}_{i}(x)\right]\right\rangle=\frac{1}{\sqrt{-\gamma}} \frac{\delta \widetilde{W}}{\delta g^{i}(x)} .
$$

Note that $\left[\mathcal{O}_{i}(x)\right]$ stands for the fully renormalized insertion of the composite operator $\mathcal{O}_{i}(x)$, which may differ from the operator monomial in an expectation value. Following JO, we make this distinction explicit by introducing the notation [...].

Using (2.14) in (2.2) and (2.4) one obtains

$$
T_{\mu}^{\mu}=\hat{\beta}^{i}\left[\mathcal{O}_{i}\right]-\mu^{-\epsilon} \beta_{\lambda} \cdot \mathscr{R}+\mu^{-\epsilon} \nabla_{\mu} \mathscr{Z}^{\mu} .
$$

This is the well-known trace anomaly, accounting for the effects of curved background and spacetime-dependent coupling constants. However, this equation is not quite correct in the most generality: there are two terms missing on the right-hand side. The first is an operator that vanishes by the equations of motion times the anomalous dimension of the corresponding quantum field. We have lost track of this term because the relation (2.1) is only correct up to terms that vanish by the equations of motion. The second missing term is more subtle: we have missed counterterms that may be needed to render some theories finite. When the kinetic terms of the Lagrangian exhibit a continuous symmetry the current associated with this symmetry is a dimension-three operator and a new type of counterterm is required in the presence of spacetime-dependent couplings, that is, a counterterm proportional to the product of the current and the derivative of a coupling. This will be discussed extensively, and the anomaly equation will be fixed, in section 3 .

Let us turn to the computation of $\mathscr{Z}^{\mu}$ in (2.4). It follows, of course, straightforwardly from the definition (2.4). Slightly less trivial is the fact that the computation must give a finite current $\mathscr{Z}^{\mu}$. That this must be so can be seen from the trace anomaly in (2.15), in which all other terms are already finite. This means that there must be cancellations among infinite terms that contribute to $\mathscr{Z}^{\mu}$. In fact, these cancellations are nothing but the consistency conditions, e.g., (2.10) and (2.12). For example, the terms in (2.4) involving the Einstein tensor (modulo terms that do not vanish for spacetime-independent $\sigma$ ) are

$$
\begin{aligned}
\int \sqrt{-\gamma} G^{\mu \nu}\left(-8 \lambda_{b} \nabla_{\mu} \partial_{\nu} \sigma-\mathscr{G}_{i j} \partial_{\mu} g^{i} \hat{\beta}^{j} \partial_{\nu} \sigma\right) & =\int \sqrt{-\gamma} \partial_{\nu} \sigma G^{\mu \nu} \partial_{\mu} g^{i}\left(8 \partial_{i} \lambda_{b}-\mathscr{G}_{i j} \hat{\beta}^{j}\right) \\
& =\int \sqrt{-\gamma} \partial_{\nu} \sigma G^{\mu \nu} \partial_{\mu} g^{i} w_{i} .
\end{aligned}
$$

Here, in going from the first to the second line we used the finiteness condition (2.12) and the definition that the finite difference is $w_{i}$. Thus we have reproduced the first term in $\mathscr{Z}_{\mu}$ of (2.6). The remaining terms in (2.6) can be similarly found. 


\subsection{Wess-Zumino action}

The Wess-Zumino action, $W_{\mathrm{WZ}}$, is some function of $\tau(x)$ that will give $-\Delta W_{\text {anomaly }}$ upon a Weyl transformation, $\tau(x) \rightarrow \tau(x)+\sigma(x)$. Focusing on the $\beta_{b}$-term in $\Delta W_{\text {anomaly }}$,

$$
-\int \sqrt{-\gamma} \sigma \beta_{b} G
$$

KS write the corresponding WZ term as ${ }^{6}$

$$
\int \sqrt{-\gamma}\left\{\tau \beta_{b} G-4 \beta_{b}\left[G^{\mu \nu} \tau_{, \mu} \tau_{, \nu}+\tau_{, \mu} \tau^{, \mu} \nabla^{2} \tau+\frac{1}{2}\left(\tau_{, \mu} \tau^{, \mu}\right)^{2}\right]\right\}
$$

where we have introduced the shorthand $\tau_{, \mu}=\partial_{\mu} \tau$. However, this computation is incomplete. The problem with this is that we have ignored the effect of the new counterterms arising from spacetime dependence of the couplings. Since we will not need a Wess-Zumino action for our generalization of the KS argument to theories with cycles, we will not aim at being complete and only point out one interesting consequence here. Consider, for example, the term in $\mathscr{Z}^{\mu}$

$$
-\int \sqrt{-\gamma} \partial_{\mu} \sigma w_{i} G^{\mu \nu} \partial_{\nu} g^{i}
$$

Now with $\partial_{\mu} g^{i}=-\beta^{i} \tau_{, \mu}$, one has the following generalization of the Wess-Zumino dilaton action:

$$
\int \sqrt{-\gamma}\left\{\beta_{b} \tau G-4\left(\beta_{b}+\frac{1}{8} w_{i} \beta^{i}\right)\left[G^{\mu \nu} \tau_{, \mu} \tau_{, \nu}+\tau_{, \mu} \tau^{, \mu} \nabla^{2} \tau+\frac{1}{2}\left(\tau_{, \mu} \tau^{, \mu}\right)^{2}\right]\right\} .
$$

The Weyl variation of (2.19) gives the sum of (2.16) and (2.18) (if $\partial_{\mu} g^{i}=-\beta^{i} \tau_{, \mu}$ there). The correction that takes $\beta_{b}$ into $\tilde{\beta}_{b}=\beta_{b}+\frac{1}{8} w_{i} \beta^{i}$ is generally of lower order than the running of $\beta_{b}$. That is, $\frac{1}{8} w_{i} \beta^{i}$ is of lower order than $\beta_{b}-\beta_{b 0}$, where $\beta_{b 0}$ stands for the free field theory value of $\beta_{b}$.

Let us be more explicit. Consider, for example, the perturbative result of JO for a pure YM theory with gauge coupling $g$,

$$
\beta_{b}=\beta_{b 0}+\frac{n_{V} \beta_{1}}{8\left(16 \pi^{2}\right)^{3}} g^{4}+\mathcal{O}\left(g^{6}\right),
$$

from which $\beta_{b}$ is seen to increase in the flow out of the trivial UV fixed point. JO also give $g w=2 n_{V} / 16 \pi^{2}+\cdots$, and therefore

$$
\tilde{\beta}_{b}=\beta_{b 0}-\frac{n_{V} \beta_{0}}{4\left(16 \pi^{2}\right)^{2}} g^{2}+\mathcal{O}\left(g^{4}\right),
$$

which shows that the leading-order running of $\tilde{\beta}_{b}$ is modified by the $\frac{1}{8} w \beta^{g}$ term. Note that $\tilde{\beta}_{b}$ decreases in the flow out of the trivial UV fixed point, as opposed to $\beta_{b}$ which, as seen from (2.20), increases. Therefore, a strong $c$-theorem in four dimensions should involve $\tilde{\beta}_{b}$, not $\beta_{b}$. Of course $\tilde{\beta}_{b}$ and $\beta_{b}$ agree at fixed points.

\footnotetext{
${ }^{6}$ The sign in the term cubic in $\tau$ is opposite to that of KS because we use the opposite sign convention for the conformal compensator, which gives $\Delta_{\sigma}$ as in JO.
} 
There is another subtle point we would like to address. The coefficient appearing in the two-point function of the trace of the stress-energy tensor appears to play the role of the "metric" $\chi_{i j}^{g}$ in the consistency condition (2.13). In appendix B we point out, following JO, that this is actually related to $-2 \chi_{i j}^{a}$, see (2.11). Explicit computations show that $-2 \chi_{i j}^{a}$ agrees with $\chi_{i j}^{g}$ to second order in perturbation theory in any four-dimensional theory. As we show in appendix B this agreement fails, for example, at third order in a YM theory with a single gauge coupling.

\section{Flavor symmetries, dimension-three operators and the corrected trace anomaly}

As we have mentioned above, in deriving the trace anomaly we have missed counterterms that may be needed to render some theories finite. When the kinetic terms of the Lagrangian exhibit a continuous symmetry the current associated with this symmetry is a dimension-three operator and a new type of counterterm is required in the presence of spacetime-dependent couplings, that is, a counterterm proportional to the product of the current and the derivative of a coupling.

Consider a theory with $n_{S}$ real scalar fields interacting through the usual quartic interaction. The kinetic part of the bare Lagrangian,

$$
\mathscr{L}_{0 K}=\frac{1}{2} \partial^{\mu} \phi_{0 a} \partial_{\mu} \phi_{0 a}
$$

exhibits a continuous symmetry under transformations of the fields $\delta \phi_{0 a}=-\omega_{a b} \phi_{0 b}$, where $\omega$ is in the algebra of the flavor group $G_{F}=\mathrm{SO}\left(n_{S}\right)$. In the process of renormalization we introduce a renormalization matrix $Z$ and write

$$
\mathscr{L}_{0 K}=\frac{1}{2} \partial^{\mu} \phi^{T} Z \partial_{\mu} \phi
$$

where renormalized fields, $\phi$, are related to bare fields by $\phi_{0}=Z^{1 / 2} \phi .{ }^{7}$ In the presence of spacetime-dependent coupling constants new divergences arise and thus new counterterms are needed. For example, one must introduce a new counterterm of the form

$$
\mathscr{L}_{\text {c.t. }}=\left(\partial^{\mu} g^{i}\right)\left(N_{i}\right)_{a b} \phi_{0 b} \partial_{\mu} \phi_{0 a},
$$

with $\left(N_{i}\right)_{a b}=-\left(N_{i}\right)_{b a}$, that is, in the algebra of $G_{F}$. Note that this new counterterm is not accounted for in $W_{\text {c.t. }}$ which by construction is independent of quantum fields. Note also that additional counterterms, symmetric under $a \leftrightarrow b$, must also be introduced. One may integrate by parts to write these as terms with no derivatives acting on the quantum fields. While necessary, they do not play a central role in what follows.

To be more explicit, we consider a theory of real scalars and write for the bare Lagrangian

$$
\mathscr{L}_{0}=\frac{1}{2} \gamma^{\mu \nu} D_{0 \mu} \phi_{0 a} D_{0 \nu} \phi_{0 a}+\frac{1}{8}(d-2) \phi_{0 a} \phi_{0 a} H-\frac{1}{4 !} g_{a b c d}^{0} \phi_{0 a} \phi_{0 b} \phi_{0 c} \phi_{0 d} .
$$

\footnotetext{
${ }^{7}$ Note that in this step we have the freedom to introduce an orthogonal matrix $O$ and define $\phi_{0}=\tilde{Z}^{1 / 2} \phi$, where $\tilde{Z}^{1 / 2}=O Z^{1 / 2}$. This does not affect $Z=Z^{T / 2} Z^{1 / 2}$. Nevertheless, such a freedom leads to an ambiguity in the definition of beta functions and anomalous dimensions as we explain in appendix A.
} 
This is written in term of bare fields $\phi_{0}$. The second term is introduced to ensure conformal invariance of the classical action. In the potential term, the bare couplings $g_{a b c d}^{0}$ are completely symmetric under exchange of the indices $a, b, c$ and $d$. The covariant derivative,

$$
D_{0 \mu} \phi_{0}=\left(\partial_{\mu}+A_{0 \mu}\right) \phi_{0}
$$

is introduced with an eye towards including the counterterm (3.1), since

$$
A_{0 \mu}=A_{\mu}+N_{I}\left(D_{\mu} g\right)_{I}, \quad D_{\mu}=\partial_{\mu}+A_{\mu}
$$

Here, following JO, we use the compact notation $I=(a b c d)$ and we have left implicit the Lie-algebra indices (so that $N_{I}^{T}=-N_{I}$ and $A_{\mu}^{T}=-A_{\mu}$ ). Note that $N_{I}$ is a function of the renormalized couplings that has an $\epsilon$-expansion starting at order $1 / \epsilon$. If the theory contains gauge fields and some of the scalars are charged under the gauge group $G_{g} \subseteq G_{F}$, it is straightforward to include an additional quantum gauge field in addition to the background field $A_{\mu}$.

The Lagrangian (3.2) is explicitly locally $G_{F}$-symmetric if we agree to transform the couplings and the gauge fields:

$$
\begin{aligned}
\delta g_{a b c d}^{0} & =-\omega_{a e} g_{e b c d}^{0}+\text { permutations } \quad\left(\delta g_{I}^{0}=-\left(\omega g^{0}\right)_{I} \text { for short }\right) \\
\delta A_{\mu} & =D_{\mu} \omega .
\end{aligned}
$$

The first of these is already used in defining the covariant derivative $\left(D_{\mu} g\right)_{I}$ in (3.3). It is very important to note at this point that if this explicit local invariance is non-anomalous it can (and will) be used to constrain the counterterms and the generating functional $\widetilde{W}$,

$$
\widetilde{W}\left[\gamma^{\mu \nu}(x),(\Omega g)_{I}(x), \Omega D_{\mu} \Omega^{-1}\right]=\widetilde{W}\left[\gamma^{\mu \nu}(x), g_{I}(x), A_{\mu}\right],
$$

where $\Omega(x)=\exp (\omega(x)) \in G_{F}$. Of course, in theories without spinors the symmetry is trivially non-anomalous. Furthermore, derivatives of the generating functional with respect to the background field now give insertions of the scalar current.

It is not our intention to repeat the calculations of JO in their entirety here. We will instead describe the main ingredients and results. We have already described the two main new ingredients, namely, the need for new counterterms and the introduction of a background field to ensure invariance under $G_{F}$ in (3.4). As before, additional quantumfield-independent counterterms are required. These are as in (2.3) but with the replacement $\partial_{\mu} \rightarrow D_{\mu}$ to ensure $G_{F}$ invariance. Additional counterterms involving the field strength $F_{\mu \nu}=\left[D_{\mu}, D_{\nu}\right]$ are also required,

$$
\tilde{\lambda} \cdot \mathscr{R}=\lambda \cdot \mathscr{R}+\frac{1}{4} \operatorname{Tr}\left(\mathscr{K} F^{\mu \nu} F_{\mu \nu}\right)+\frac{1}{2} \operatorname{Tr}\left(\mathscr{P}_{I J} F^{\mu \nu}\right)\left(D_{\mu} g\right)_{I}\left(D_{\nu} g\right)_{J}
$$

Moreover, as advertised, new field-dependent counterterms must be included,

$$
\mathscr{Q}=\eta_{a b} \phi_{a} \phi_{b} H+\left(\delta_{I}\right)_{a b} \phi_{a} \phi_{b}\left(D^{2} g\right)_{I}+\frac{1}{2}\left(\epsilon_{I J}\right)_{a b} \phi_{a} \phi_{b}\left(D^{\mu} g\right)_{I}\left(D_{\mu} g\right)_{J}
$$


Proceeding much as before, JO find ${ }^{8}$ [8, Eq. (6.15)]

$$
T_{\mu}^{\mu}=\hat{\beta}_{I}\left[\mathcal{O}_{I}\right]+\left[\beta^{\mathscr{Q}}\right]+\left[\left(D^{\mu} \phi\right)^{T} \beta_{\mu}^{A} \phi\right]-\mu^{-\epsilon} \beta_{\tilde{\lambda}} \cdot \mathscr{R}+\nabla_{\mu}\left(J^{\mu}+J_{\Theta}^{\mu}+\widetilde{\mathscr{Z}^{\mu}}\right)-((1+\hat{\gamma}) \phi) \cdot \frac{\delta}{\delta \phi} \tilde{S}_{0},
$$

which, using the underlying gauge invariance, they rewrite as [8, Eq. (6.23)]

$$
T_{\mu}^{\mu}=\widehat{B}_{I}\left[\mathcal{O}_{I}\right]+\left[\beta^{\mathscr{Q}}\right]+\left[\left(D_{\mu} \phi\right)^{T} B_{\mu}^{A} \phi\right]-\mu^{-\epsilon} \beta_{\tilde{\lambda}} \cdot \mathscr{R}+\nabla_{\mu}\left(J_{\Theta}^{\mu}+\widetilde{\mathscr{Z}^{\mu}}\right)-((1+\hat{\gamma}+S) \phi) \cdot \frac{\delta}{\delta \phi} \tilde{S}_{0}
$$

Many comments are in order. The last term, involving the derivative of the full action integral $\tilde{S}_{0}$, vanishes by the equations of motion. We have included it here for completeness. We have already commented that a similar term is missing from (2.15). The operator $\left[\mathcal{O}_{I}\right]$ corresponds to the interaction term in the Lagrangian, $\mathcal{O}_{I}=\frac{1}{4 !} \phi_{a} \phi_{b} \phi_{c} \phi_{d}$, but differs from it, $\left[\mathcal{O}_{I}\right]=\mathcal{O}_{I}-\nabla_{\mu} J_{I}^{\mu}$ where $J_{I}^{\mu}=\left(D_{0}^{\mu} \phi_{0}\right)^{T} N_{I} \phi_{0}$. Its coefficient in (3.8) is given by

$$
\widehat{B}_{I} \equiv \hat{\beta}_{I}-(S g)_{I},
$$

where $\hat{\beta}_{I}=\mu d g_{I} / d \mu=-\epsilon g_{I}+\beta_{I}$. The current $J^{\mu}$ in (3.7) is defined as

$$
J^{\mu}=\left(D_{0}^{\mu} \phi_{0}\right)^{T} N_{I} \hat{\beta}_{I} \phi_{0}
$$

and it is finite as required from consistency of (3.7). Note that the combination $\left[\mathcal{O}_{I}\right]+\nabla_{\mu} J_{I}^{\mu}$ appearing in (3.7) is just $\mathcal{O}_{I}$. However, $\mathcal{O}_{I}$ is not by itself a finite operator. While $\hat{\beta}_{I} \mathcal{O}_{I}$ is finite, since it is the sum of two finite operators, replacing $\hat{\beta}_{I}$ by its $\epsilon \rightarrow 0$ limit, $\beta_{I} \mathcal{O}_{I}$, is not by itself finite. Finiteness of $J^{\mu}$ implies that it can be brought to the form

$$
J^{\mu}=\left[\left(D^{\mu} \phi\right)^{T} S \phi\right] .
$$

The Lie-algebra element $S$ is then defined by $\widehat{B}_{I} N_{I}=S$. Since $S$ is finite it is required that the infinite pieces of $\widehat{B}_{I} N_{I}$ cancel automatically, i.e.,

$$
\widehat{B}_{I} N_{I}=S \Rightarrow S=-N_{I}^{1} g_{I},
$$

where $N_{I}=\sum_{n=1}^{\infty} N_{I}^{n} / \epsilon^{n}$, so that $N_{I}^{1}$ is the residue of the simple $\epsilon$-pole in $N_{I}$. Cancellation of the infinite pieces requires that $B_{I} N_{I}^{n}-g_{I} N_{I}^{n+1}=0$ for $n \geq 1$. The beta functions for the field-dependent quadratic counterterms are

$$
\beta^{\mathscr{Q}} \equiv \beta_{a b}^{\eta} \phi_{a} \phi_{b} H+\left(\beta_{I}^{\delta}\right)_{a b} \phi_{a} \phi_{b}\left(D^{2} g\right)_{I}+\frac{1}{2}\left(\beta_{I J}^{\epsilon}\right)_{a b} \phi_{a} \phi_{b}\left(D_{\mu} g\right)_{I}\left(D_{\mu} g\right)_{J} .
$$

The term $\beta_{\tilde{\lambda}} \cdot \mathscr{R}$ is the obvious generalization of (2.5) while the current $\widetilde{\mathscr{Z}}^{\mu}$ is defined as in (2.6) but rendered $G_{F}$-invariant by replacing derivatives by covariant derivatives. In addition, $\widetilde{\mathscr{Z}^{\mu}}$ has contributions from the new counterterms in (3.5), and there are additional contributions to the terms with the $\mathscr{A}$ and $\mathscr{B}$ of (2.3). The third term in (3.8) involves

$$
B_{\mu}^{A} \equiv \beta_{\mu}^{A}+D_{\mu} S \equiv \rho_{I}\left(D_{\mu} g\right)_{I}+D_{\mu} S \equiv P_{I}\left(D_{\mu} g\right)_{I}, \quad \rho_{I}=g_{J} \partial_{J} N_{I}^{1}+N_{I}^{1},
$$

\footnotetext{
${ }^{8}$ Note that in Jack and Osborn the first term contains $\hat{\beta}^{V}=\mu d V / d \mu$, where $V$ is the renormalized potential, and with the derivative taken by holding the bare fields, $\phi_{0}$, and the bare potential, $V_{0}$, constant (independent of RG time). With a potential of the form $V=g_{I} \mathcal{O}_{I}$ and following Jack and Osborn's definitions we then obtain $\left[\hat{\beta}^{V}\right]=\hat{\beta}_{I}\left[\mathcal{O}_{I}\right]$ as expected.
} 
where $\beta_{\mu}^{A} \equiv \mu d A_{\mu} / d \mu$ is the beta function for the background gauge field $A_{\mu}$. Finally, the current $J_{\Theta}^{\mu}$ arises from the counterterms in (3.6) and has a complicated expression in terms of the simple $\epsilon$-poles in $\delta_{I}$ and $\epsilon_{I J}$ (see JO for details [8, Eqs. (6.21-22)]).

At this point we can take the limit of flat spacetime, spacetime-independent couplings and no background gauge field in (3.8). This gives

$$
T_{\mu}^{\mu}=\widehat{B}_{I}\left[\mathcal{O}_{I}\right]-((1+\hat{\gamma}+S) \phi) \cdot \frac{\delta}{\delta \phi} S_{0} .
$$

Since $\left[\mathcal{O}_{I}\right]$ is finite we can now safely conclude that a theory is conformal if and only if $B_{I}=0$. This does not require that $\beta_{I}=0$.

In the general case considered here the JO consistency conditions are modified relative to what has been presented in section 2. On the one hand the conditions have to be covariant under transformations by the symmetry group $G_{F}$. On the other, there are additional terms that arise from the additional counterterms required to render the theory finite. Osborn gives the form of these most general consistency conditions [14]. Two conditions play a role in our discussion:

$$
\begin{aligned}
8 \partial_{I} \beta_{b} & =\chi_{I J}^{g} B_{J}-B_{J} \partial_{J} w_{I}-\left(\partial_{I} B_{J}\right) w_{J}-\left(P_{I} g\right)_{J} w_{J} \\
& =\chi_{I J}^{g} B_{J}-\beta_{J} \partial_{J} w_{I}-\left(\partial_{I} \beta_{J}\right) w_{J}-\left(\rho_{I} g\right)_{J} w_{J}
\end{aligned}
$$

and

$$
B_{I} P_{I}=0 .
$$

In addition, covariance under $G_{F}$ gives, e.g.,

$$
(\omega g)_{I} \partial_{I} \beta_{b}=0 \quad \text { and } \quad(\omega g)_{I} \partial_{I} S=[\omega, S] .
$$

Of course, the first of these applies to any $G_{F}$-invariant while the second to any antisymmetric tensor (for example, any Lie-algebra valued function). Using the first of (3.15) in (3.13) gives a nontrivial relation among several beta functions:

$$
(\omega g)_{I}\left[\chi_{I J}^{g} B_{J}-B_{J} \partial_{J} w_{I}-\left(\partial_{I} B_{J}\right) w_{J}-\left(P_{I} g\right)_{J} w_{J}\right]=0,
$$

or, equivalently,

$$
(\omega g)_{I}\left[\chi_{I J}^{g} B_{J}-\beta_{J} \partial_{J} w_{I}-\left(\partial_{I} \beta_{J}\right) w_{J}-\left(\rho_{I} g\right)_{J} w_{J}\right]=0 .
$$

These conditions can be used to understand aspects of the flow of $\beta_{b}$. Consider the flow defined by some arbitrary function $f_{I}(g)$,

$$
\frac{d \bar{g}_{I}}{d \eta}=-f_{I}(\bar{g}(\eta))
$$

If one takes $f_{I}=\beta_{I}$ then the flow can be identified with the RG flow, with $\eta=t=$ $-\ln \left(\mu / \mu_{0}\right)$. From (3.13) we have

$$
-8 \frac{d \widetilde{B}_{b}}{d \eta}=\chi_{I J}^{g} f_{I} B_{J}+f_{I} B_{J} \partial_{[I} w_{J]}-\left(P_{I} g\right)_{J} f_{I} w_{J},
$$


where

$$
\widetilde{B}_{b}=\beta_{b}+\frac{1}{8} B_{I} w_{I}
$$

and

$$
-8 \frac{d \tilde{\beta}_{b}}{d \eta}=\chi_{I J}^{g} f_{I} B_{J}+f_{I} \beta_{J} \partial_{[I} w_{J]}-\left(\rho_{I} g\right)_{J} f_{I} w_{J} .
$$

Three special cases are of most interest. Consider first $f_{I}(g)=-(\omega g)_{I}$. From the second equation in (3.15) we see that on this flow $\omega$ is constant. This is a recursive flow (cycle or ergodic). It follows from the $G_{F}$-invariance of $\widetilde{B}_{b}$ and $\tilde{\beta}_{b}$ that these remain constant on the flow. This is a consequence of the detailed cancellations that must be satisfied by the beta functions in (3.16) and (3.17). This general result can be applied to limit cycles, $\beta_{I}=(Q g)_{I}$, for which $\omega=Q$. We thus see that counterterms that ensure $G_{F}$-covariance guarantee constancy of $\beta_{b}$ (and $\tilde{\beta}_{b}$ ) on recursive flows.

The second and third special cases correspond to $f_{I}=B_{I}$ and $f_{I}=\beta_{I}$. While the $B_{I}$ and $\beta_{I}$ flows are generally distinguishable, one may use (3.15) to show the two flows are identical for $G_{F}$-invariants. ${ }^{9}$ Using (3.18) with $f_{I}=B_{I}$ and the consistency condition (3.14) we see that

$$
-8 \frac{d \widetilde{B}_{b}}{d t}=\chi_{I J}^{g} B_{I} B_{J}
$$

This shows that $\widetilde{B}_{b}$ decreases monotonically along both flows and is a good candidate for the $c$ function of the $c$-theorem. Indeed this shows a strong version of the $c$-theorem in perturbation theory. To two loops $\chi_{I J}^{g}=-2 \chi_{I J}^{a}>0$, where unitarity is required for the inequality, so the right-hand side of (3.21) is positive-definite along a perturbative flow.

The relation between the $B_{I}$ and $\beta_{I}$ flows can be made more explicit, hopefully clarifying their relation. Consider the flows

$$
-\frac{d g_{I}}{d t}=\beta_{I}(g(t)) \quad \text { and } \quad-\frac{d \bar{g}_{I}}{d \eta}=B_{I}(\bar{g}(\eta)) .
$$

The solution to the $\eta$-flow is given in terms of the one for the RG flow by

$$
\bar{g}(\eta)=F(\eta) g(\eta) \quad \text { where } \quad F(\eta)=T\left(\exp \left[-\int_{-\infty}^{\eta} d \eta^{\prime} S\left(\eta^{\prime}\right)\right]\right) .
$$

Here $T$ is the $\eta$-ordered product and $F \in G_{F}$. As such, $\beta_{I}(\bar{g})=\beta_{I}(F g)=(F \beta)_{I}(g)$ and similarly for $B_{I}$ and indeed for any tensor function of the couplings. Of special interest are $G_{F}$-invariants, like $\widetilde{B}_{b}$, for which $\widetilde{B}_{b}(\bar{g})=\widetilde{B}_{b}(F g)=\widetilde{B}_{b}(g)$. So we see again that the monotonic $\eta$-flow of $\widetilde{B}_{b}$ gives a monotonic RG flow of $\widetilde{B}_{b}$.

The quantity $\tilde{\beta}_{b}$ does not appear to be a good candidate for the $c$ function of the $c$-theorem. Using (3.20) to study its flow, so the term $f_{I} \beta_{J} \partial_{[I} w_{J]}$ automatically vanishes, we obtain

$$
-8 \frac{d \tilde{\beta}_{b}}{d t}=\chi_{I J}^{g} \beta_{I} B_{J}-\left(\rho_{I} g\right)_{J} \beta_{I} w_{J} .
$$

Were we to ignore the last term on the right-hand side we would be able to establish a perturbative $c$-theorem for $\tilde{\beta}_{b}$. Indeed, to two loops $B_{I}=\beta_{I}$ and $\chi_{I J}^{g}=-2 \chi_{I J}^{a}>0$ so the

\footnotetext{
${ }^{9}$ This was pointed out to us by J. Polchinski.
} 
right-hand side of (3.22) would be positive-definite along a perturbative flow. However, the last term is parametrically of the same order as the first on the right-hand side of (3.22) so this does not give a perturbative $c$-theorem for $\tilde{\beta}_{b}$.

\section{Scale implies conformal invariance}

\section{1 $S$ is $Q$ (on cycles)}

In this subsection we elucidate the relation between $Q$ and $S$. Our treatment is focused on theories in $d=4$. We remind the reader that $Q$ is defined as the solution to the equations $\beta^{g}=0$ and $\beta_{I}=(Q g)_{I}$, defining an RG cycle on which $Q$ remains a constant while $S$ is defined as a function of couplings that makes explicit the finiteness of the current $J^{\mu}$ in (3.10). There is no a priori reason they should be related.

What is known about $S$ ? JO have shown, by direct calculation, and we have verified, that in a scalar field theory $S$ vanishes up to third order in the loop expansion. The result holds even if gauge fields are included and the scalars are charged under the gauge group. For theories with scalars and fermions, JO have shown, and we have verified, that $S$ remains zero to two loops. However, this is consistent with a possible equality of $S$ and $Q$ on cycles. Indeed, we have obtained previously that $Q$ is of third order in the loop expansion in Yang-Mills theories with scalars and fermions, while in purely-scalar field theories a non-vanishing $Q$, if it exists, must be at least of fifth order in the loop expansion.

As might be expected from the discussion above, we will show that (up to conserved current)

1. $S$ is $Q$ on cycles,

2. $S$ vanishes at fixed points.

In light of these results the computation of $Q$ can be tremendously simplified given an explicit expression for $S$. Presently, the procedure to determine $Q$ involves determining first the beta functions for the coupling constants to second order in the loop expansion for scalar self-couplings, to third order in the loop expansion for Yukawa couplings and to fourth order in the loop expansion for Yang-Mills couplings, and then solving the system of nonlinear coupled equations $\beta^{g}=0$ and $\beta_{I}=(Q g)_{I}$ (we implicitly use here that $g_{I}$ can also stand for Yukawa couplings). Since $S$ must have a perturbative expansion that starts at third order in the loop expansion, to determine $Q$ from $S$ it suffices to evaluate it with coupling constants on the cycle computed to lowest order in the loop expansion. So $Q$ is obtained from $S$ by determining the zeroes of the one-loop beta functions (two-loop for gauge couplings): if $S=0$ on the zero of the beta functions, the zero is a fixed point of the RGE, but if $S \neq 0$ on the zero, then the zero is a point on a cycle and $Q=S$ there.

To this end an explicit, three-loop expression for $S$ is required. But as pointed out above, there has been no computation of $S$ to the order where one would expect it to be non-vanishing if $S$ were to equal $Q$ on cycles. We have endeavored to compute $S$ to third order in the loop expansion for a general theory containing $n_{S}$ real scalars and $n_{f}$ Weyl 
spinors, possibly charged under a gauge group. The potential in the Lagrangian is

$$
V=\frac{1}{4 !} \lambda_{a b c d} \phi_{a} \phi_{b} \phi_{c} \phi_{d}+\left(\frac{1}{2} y_{a \mid i j} \phi_{a} \psi_{i} \psi_{j}+\text { h.c. }\right) \text {. }
$$

The details of the computation are spelled out in appendix C. The surprisingly simple result is

$$
\left(16 \pi^{2}\right)^{3} S_{a b}=\frac{5}{8} \operatorname{tr}\left(y_{a} y_{c}^{*} y_{d} y_{e}^{*}\right) \lambda_{b c d e}+\frac{3}{8} \operatorname{tr}\left(y_{a} y_{c}^{*} y_{d} y_{d}^{*} y_{b} y_{c}^{*}\right)-\{a \leftrightarrow b\}+\text { h.c.. }
$$

We have evaluated this expression on the fixed points and cycles of the theories we explored in $[4,6,7]$ and found that in each case, even in examples in $d=4-\epsilon, S$ vanishes at all fixed points and equals our previous determination of $Q$ on all cycles.

Now for the (perturbative) proof of the propositions above. First we show that $S=Q$ on cycles. Consider the $\eta$-flow with $f_{I}=B_{I}$, with boundary condition that at $\eta=0$ the point $\bar{g}_{I}(0)$ is on the cycle. Then $B_{I}(0)=\beta_{I}(\bar{g}(0))-(S g(0))_{I}=([Q-S] g(0))_{I}$, with $Q-S$ in the Lie algebra of $G_{F}$ and the left-hand side of (3.21) vanishes. Since $\chi_{I J}^{g}$ is positive-definite to second order in the loop expansion, $(3.21)$ gives $B_{I}(0)=0$. This implies $S=Q+\Delta Q$ on cycles, where $(\Delta Q g)_{I}=0$. But if $\Delta Q \neq 0$ this corresponds to a conserved current, $\nabla_{\mu}\left[\left(D^{\mu} \phi\right)^{T} \Delta Q \phi\right]=0$, and we are free to redefine the scale current by a conserved current by $Q \rightarrow Q+\Delta Q$. Hence, $S=Q$ on cycles. ${ }^{10}$

For theories with two scalars there is an alternative, perhaps simpler proof that $S$ equals $Q$ when evaluated on a cycle. Consider (3.21) evaluated on a point on the cycle. It is easy to show that $S$ is a constant on the cycle: $-d S / d t=\beta_{I} \partial_{I} S=(Q g)_{I} \partial_{I} S=[Q, S]=0$, where the last two steps follow from (3.15) and the fact that, for two flavors, the flavor group, $\mathrm{SO}(2)$, is Abelian. Now, as before, we consider the $\eta$-flow defined by the $B_{I}$ function starting from a point on the RG-cycle (we make the distinction of the actual RG-cycle and a $\eta$-cycle explicit, to avoid confusion). The flow is defined by $-d \bar{g}_{I} / d \eta=B_{I}=\beta_{I}-(S g)_{I}=$ $([Q-S] g)_{I}$, where the last step follows from assuming the initial point is on the RG-cycle and then noting that the solution corresponds to a trajectory that traverses the same cycle but at a different angular speed (the angular speeds are $Q_{12}$ and $Q_{12}-S_{12}$ for the RGand $\eta$-cycles, respectively). Therefore the $\eta$-cycle is generated by a trajectory in $G_{F}$ and it follows that, just as for an RG-cycle, any $G_{F}$-invariant remains constant on the $\eta$-cycle. But the consistency condition (3.21) then implies that $B_{I}=([Q-S] g)_{I}=0$ on the cycle. Since $Q$ and $S$ are each characterized by a single number the only solution is $S_{12}=Q_{12}$ (on the cycle).

It is easy to show that $(S g)_{I}=0$ at a fixed point, and this is consistent with the notion that a fixed point corresponds to the case $(Q g)_{I}=0$. To see this, notice that at a fixed point $B_{I}=-(S g)_{I}$ so at that point the flow corresponds to a first-order $G_{F}$-transformation. That is, the first derivative with respect to $\eta$ of $G_{F}$-invariants vanishes at the fixed point. Hence, (3.21) gives that $\chi_{I J}^{g}(S g)_{I}(S g)_{J}=0$ and hence $(S g)_{I}=0$ at the fixed point. The solution is that either $S=0$ at the fixed point, or there is an emergent symmetry at the

\footnotetext{
${ }^{10}$ In unitary theories with $\mathcal{N}=1$ supersymmetry we recently showed, without relying on perturbation theory, that $S=0$ [17]. It thus follows that RG limit cycles do not arise in such theories.
} 
fixed point, and $J^{\mu}$ is the corresponding conserved current. This completes the proof of the two propositions above.

\subsection{Cyclic CFTs}

\subsubsection{A perturbative proof that scale imples conformal invariance}

The condition for a theory in $d>2$ to be scale-invariant is that the trace of its stress-energy tensor be a total derivative [9],

$$
T_{\mu}^{\mu}=\partial_{\mu} V^{\mu}
$$

where $V^{\mu} \neq j^{\mu}+\partial_{\nu} L^{\mu \nu}$ with $\partial_{\mu} j^{\mu}=0$ and, without loss of generality, $L^{\mu \nu}=L^{\nu \mu}$. A candidate for $V^{\mu}$ is $V^{\mu}=\partial^{\mu} \phi^{T} P \phi$. If the theory includes spinors an additional current can be added to $V^{\mu}$ but the argument below is easily generalized by trivial extensions, e.g., by interpreting the index $I$ as including all couplings. Using the equations of motion, or alternatively a $G_{F}$-transformation, this can be cast as an algebraic condition,

$$
B_{I}=(P g)_{I}
$$

It is easy to see now that in $d=4$ the $B_{I}$-flow of $\widetilde{B}_{b}$ requires $(P g)_{I}=0$. Indeed, using (4.1) in (3.21) the left-hand side vanishes on account of $B_{I}$ being of the form $(\omega g)_{I}$, and then perturbative positivity of $\chi_{I J}^{g}$ implies $B_{I}=0$. While $P$ may not vanish, the current $V^{\mu}$ can at most be a symmetry of the theory, $V^{\mu}=j^{\mu}$. This concludes the proof that scale implies conformal invariance in perturbation theory.

\subsubsection{Some properties of cyclic CFTs}

Our result that scale implies conformal invariance implies that the non-trivial cycle found in [7] actually corresponds to a CFT. We dub such CFTs cyclic CFTs. It is quite surprising that CFTs can be found at points where the beta functions do not vanish. It is unclear what, if anything, distinguishes these theories from fixed-point CFTs. Presumably the special current $J^{\mu}$ plays a crucial role. We hope to address these questions in the future, but at present have no progress to report.

Since the stress-energy tensor is not renormalized, and since the divergence of the special current $J^{\mu}$ appears in the trace-anomaly equation, one may suspect its anomalous dimension vanishes. If so this would correspond to a non-conserved vector operator of dimension exactly three (no anomalous dimension), which is impossible in a unitary CFT. However, the operator actually mixes under renormalization. A simple computation gives

$$
\begin{aligned}
\mu \frac{d}{d \mu}\left[\mathcal{O}_{I}\right] & =-\partial_{I} \hat{\beta}_{J}\left[\mathcal{O}_{J}\right]+\partial_{\mu}\left[\partial^{\mu} \phi^{T} \rho_{I} \phi\right], \\
\mu \frac{d}{d \mu}\left[\partial^{\mu} \phi^{T} \omega \phi\right] & =-\left[\partial^{\mu} \phi^{T} \rho_{I}(\omega g)_{I} \phi\right],
\end{aligned}
$$

which allows one to readily verify that (i) the combination $\hat{\beta}_{I}\left[\mathcal{O}_{I}\right]+\partial_{\mu} J^{\mu}$ is RG-invariant, (ii) a symmetry current is RG-invariant, and (iii) $J^{\mu}$ is not RG-invariant, $\mu \frac{d}{d \mu} J^{\mu}=$ $-\left[\partial^{\mu} \phi^{T} \hat{\beta}_{I} \rho_{I} \phi\right]$. 
Even if the beta function is non-vanishing, properties that follow directly from the conformal symmetry apply to these cyclic CFTs. Consider for example the well-known fact that two point correlators of primary operators can be diagonalized and

$$
\left\langle\mathcal{O}(x)^{\dagger} \mathcal{O}(0)\right\rangle=\left(x^{2}\right)^{-\Delta_{\mathcal{O}}}
$$

Now contrast this with the two point function of the elementary real scalars $\phi_{a}$ in a cyclic CFT. Scale and Poincaré invariance alone give [5]

$$
\left\langle\phi(x) \phi^{T}(0)\right\rangle=\left(x^{2}\right)^{-\frac{1}{2} \Delta} G\left(x^{2}\right)^{-\frac{1}{2} \Delta^{T}},
$$

where $G$ is a fixed real, positive, symmetric matrix and $\Delta=1+\gamma+Q$, with $\gamma^{T}=\gamma$ the anomalous dimension matrix of the elementary fields $\phi$ and $Q^{T}=-Q$ defining the cycle through $\beta_{I}=(Q g)_{I}$. Now one can redefine the field by $\phi \rightarrow M^{-1} \phi$ with $M$ chosen so that $M G M^{T}=1$, which is always possible with real $M$ for a real, positive, symmetric matrix. This effectively redefines $\Delta \rightarrow M \Delta M^{-1}$. The condition for invariance under special conformal transformations then gives ${ }^{11} \Delta^{T}=\Delta$. A further field redefinition by an appropriate orthogonal transformation $R$ finally brings $\Delta$ into diagonal form, $\Delta \rightarrow$ $R \Delta R^{T}$. The entries of this diagonal form of $\Delta$ correspond to the roots of the characteristic polynomial of $1+\gamma+Q$ which must be real. It is interesting that this puts restrictions on the possible values of $Q$ : given a fixed value of $\gamma$, for large enough $Q$ some roots will be complex. To put it differently, from our proof that these theories are conformally invariant we infer that if a matrix $X A X^{-1}$ is diagonal for a real matrix $A$ and a real, symmetric, invertible matrix $X$, then all the roots of the characteristic polynomial of $A$ are real.

This unfortunately means that the large- $Q$ scenario of [5], which leads to interesting oscillatory behavior in unparticle physics, is excluded by conformal invariance. More generally, the constraints that unitarity and scale invariance alone place on the scaling dimensions of operators are weaker than those that follow if conformal invariance is also imposed [18]. These weaker conditions are popular in unparticle phenomenology as they amplify the putative effects of unparticles. Of course our proof does not rule out theories that are scale-invariant but not conformal outside the realm of perturbation theory, leaving a smidgen of hope for unparticle enthusiasts.

\section{The $c$-theorem in the presence of cycles}

As we have seen, the consistency relations of JO lead to the $c$-theorem in perturbation theory,

$$
-\frac{d \widetilde{B}_{b}}{d t}=\frac{1}{8} \chi_{I J}^{g} B_{I} B_{J} \geq 0
$$

with $\widetilde{B}_{b}$ defined in (3.19). Only the last step in this sequence of relations invokes perturbation theory, for the positivity of the metric $\chi_{i j}^{g}$ is established perturbatively. For a non-perturbative proof we turn to the method of KS.

\footnotetext{
${ }^{11}$ Alternatively, special conformal transformations on (4.2) require that $\Delta G=G \Delta^{T}$.
} 
Let us review the argument of KS. Our presentation is closer in spirit to that of LPR. We will try to note explicitly when implicit assumptions in that argument are made. While plausible, these assumptions should be justified for the theorem to be established. We deviate from both presentations in that we do not derive nor use a Wess-Zumino dilaton action for, as we will see, this is not necessary for the computation.

Consider the four point function of the operator $\frac{1}{2} \partial_{\mu}\left(x_{\nu} T^{\mu \nu}\right)$ in an arbitrary fourdimensional theory which is classically scale-invariant. Furthermore, we will consider kinematics such that $p_{i}^{2}=0, i=1, \ldots, 4$, for the momenta $p_{i}$ of the four insertions, so that the Mandelstam variables satisfy $s+t+u=0$. Equivalently, for the theory on a conformally flat background, $g_{\mu \nu}=e^{-2 \tau(x)} \eta_{\mu \nu}$, one may compute the $\tau(x)$ scattering amplitude $A(s, t)$ with the on-shell condition $\nabla^{2} \tau=0$.

Now, we will assume that the forward scattering amplitude $\mathcal{A}_{\mathrm{fwd}}(s)=\mathcal{A}(s, 0)$ exists, that is that the limit $t \rightarrow 0$ of $\mathcal{A}(s, t)$ exists. This could fail if $\mathcal{A}(s, t)$ had terms of the form, e.g., $\sim s^{2} \ln (t)$. Now, $\mathcal{A}_{\mathrm{fwd}}(s)$ can be computed by taking four $\tau(x)$-derivatives of the generating functional and then taking the metric as flat, the coupling constants to be spacetimeindependent and the background field $A_{\mu}$ and the conformal compensator to vanish. Alternatively, and more straightforwardly, one can work with a conformally flat metric and having the only spacetime dependence in couplings and $A_{\mu}$ arise through the dependence on the conformal factor $\tau(x)$, so that one merely needs to take $\tau(x)=0$ after four times differentiating $\widetilde{W}$. Now the first derivative simply gives the conformal anomaly equation

$$
T_{\mu}^{\mu}=\widehat{B}_{I}\left[\mathcal{O}_{I}\right]+\left[\beta^{\mathscr{Q}}\right]+\left[\left(D^{\mu} \phi\right)^{T} B_{\mu}^{A} \phi\right]-\mu^{-\epsilon} \beta_{\tilde{\lambda}} \cdot \mathscr{R}+\nabla_{\mu}\left(J_{\Theta}^{\mu}+\widetilde{\mathscr{Z}^{\mu}}\right)-((1+\hat{\gamma}+S) \phi) \cdot \frac{\delta}{\delta \phi} \tilde{S}_{0},
$$

One need only thrice differentiate this equation to obtain the four-point amplitude of $T_{\mu}^{\mu}$. Note that on fixed points and cycles, where we will need this, the first term vanishes since $\widehat{B}_{I}=0$. Also, the last term, which vanishes by the equations of motion, can be ignored for the computation of the amplitude. Most of the remaining terms vanish once the couplings are taken to be spacetime-independent (and the metric flat and $A_{\mu}=0$ ). The remaining terms arise from the $G$ and $H^{2}$ terms in $\beta_{\lambda} \cdot \mathscr{R}$. For a conformally flat metric, $\gamma_{\mu \nu}=\exp (-2 \tau(x)) \eta_{\mu \nu}$, one has (in $d$ spacetime dimensions)

$$
\begin{aligned}
e^{-4 \tau} G & =8\left(\partial^{2} \tau\right)^{2}-8 \tau_{, \mu \nu} \tau^{, \mu \nu}-16 \tau_{, \mu} \tau_{, \nu} \tau^{, \mu \nu}-8(d-3) \tau_{, \mu} \tau^{, \mu} \partial^{2} \tau+2(d-1)(d-4)\left(\tau_{, \mu} \tau^{, \mu}\right)^{2} \\
e^{-4 \tau} H^{2} & =4\left(\partial^{2} \tau\right)^{2}-4(d-2) \tau_{, \mu} \tau^{, \mu} \partial^{2} \tau+(d-2)^{2}\left(\tau_{, \mu} \tau^{, \mu}\right)^{2} .
\end{aligned}
$$

The cubic term in $H^{2}$ vanishes for an "on-shell" conformal factor $\partial^{2} \tau=0$ and so the only contribution to the "on-shell" forward scattering amplitude is from $G$ :

$$
\left.\mathcal{A}_{\mathrm{fwd}}(s)\right|_{\mathrm{FP} \text { or cycle }}=-\left.\beta_{b}\left(s^{2}+t^{2}+u^{2}\right)\right|_{t=0}=-2 \beta_{b} s^{2} .
$$

Let's assume that there exists an RG trajectory from a UV fixed point or cycle to an IR fixed point or cycle. On this trajectory this equation no longer holds. However, we can inspect limiting behavior. Since $\mathcal{A}_{\mathrm{fwd}} / s^{2}$ depends on $s$ only through the dimensionless ratio $\mu^{2} / s$, its behavior is dictated by the renormalization group. Hence,

$$
\lim _{s \rightarrow \infty} \frac{\mathcal{A}_{\mathrm{fwd}}(s)}{s^{2}}=\lim _{s \rightarrow \infty} \frac{\left.\mathcal{A}_{\mathrm{fwd}}(s)\right|_{\mathrm{FP} \text { or cycle }}}{s^{2}}=-2\left(\beta_{b}\right)_{\mathrm{UV}}
$$




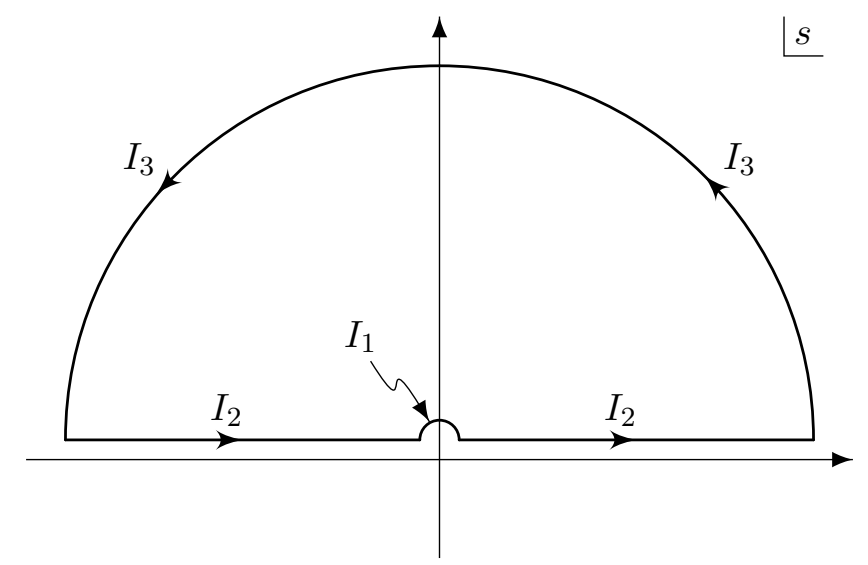

Figure 1. The contour of integration for $\int \frac{d s}{s^{3}} \mathcal{A}_{\mathrm{fwd}}(s)$.

and

$$
\lim _{s \rightarrow 0} \frac{\mathcal{A}_{\mathrm{fwd}}(s)}{s^{2}}=\lim _{s \rightarrow 0} \frac{\left.\mathcal{A}_{\mathrm{fwd}}(s)\right|_{\mathrm{FP} \text { or cycle }}}{s^{2}}=-2\left(\beta_{b}\right)_{\mathrm{IR}},
$$

where $\left(\beta_{b}\right)_{\mathrm{UV}}$ and $\left(\beta_{b}\right)_{\mathrm{IR}}$ are the limiting UV and IR values of $\beta_{b}$ along the trajectory and correspond to those on the fixed point or cycle. LPR study the approach to these limiting values using conformal perturbation theory.

Following LPR we next consider the integral of $\mathcal{A}_{\mathrm{fwd}}(s) / s^{3}$ over the contour in figure 1 . The integral over the semicircle $I_{1}$ cannot be easily computed, but in the limit that the radius of the semicircle vanishes it is reasonable that one can use the limiting value,

$$
\int_{I_{1}} \frac{d s}{s^{3}} \mathcal{A}_{\mathrm{fwd}}(s) \approx \int_{I_{1}} \frac{d s}{s} 2\left(\beta_{b}\right)_{\mathrm{IR}}=2 \pi i\left(\beta_{b}\right)_{\mathrm{IR}},
$$

where the last step corresponds to taking the vanishing limit of the radius of the semicircle $I_{1}$. Similarly, the large circle $I_{3}$ gives

$$
\int_{I_{3}} \frac{d s}{s^{3}} \mathcal{A}_{\mathrm{fwd}}(s) \approx \int_{I_{3}} \frac{d s}{s} 2\left(\beta_{b}\right)_{\mathrm{UV}}=-2 \pi i\left(\beta_{b}\right)_{\mathrm{UV}} .
$$

It follows from Cauchy's theorem that

$$
\begin{aligned}
\left(\beta_{b}\right)_{\mathrm{UV}}-\left(\beta_{b}\right)_{\mathrm{IR}} & =\frac{1}{2 \pi i} \int_{I_{2}} \frac{d s}{s^{3}} \mathcal{A}_{\mathrm{fwd}}(s) \\
& =\frac{1}{\pi} \int_{0}^{\infty} \frac{d s}{s^{3}} \operatorname{Im}\left(\mathcal{A}_{\mathrm{fwd}}(s+i 0)\right),
\end{aligned}
$$

where in the last line LPR assume crossing symmetry to write $\mathcal{A}_{\mathrm{fwd}}(-s+i 0)=\mathcal{A}_{\mathrm{fwd}}^{*}(s+i 0)$. Finally, the KS argument invokes the optical theorem that relates the imaginary part of the forward scattering amplitude to a positive-definite cross section to conclude that

$$
\left(\beta_{b}\right)_{\mathrm{UV}}-\left(\beta_{b}\right)_{\mathrm{IR}}>0 .
$$

We note in passing that the optical theorem is known to apply for forward scattering amplitudes of (on-shell) physical particles. It is not clear a priori that it applies to Green 
functions of composite operators at $p_{i}^{2}=0$, even if it corresponds to the scattering amplitude of would-be dilaton scattering. We think the assumption of positivity is reasonable, so we press on.

What steps in the argument above require special attention when the theory admits dimension-three currents? As we have pointed out, the trace of the stress-energy tensor now has an additional $\partial_{\mu} J^{\mu}$ term, but we have already accounted for this in the presentation above: the current can be eliminated by replacing $B_{I}$ for $\beta_{I}$ in the expression for the trace of the stress-energy tensor. Throughout the flow this makes no difference to the argument above, since the positivity of the integral over the segments $I_{2}$ of the contour follows from the optical theorem. For cycles one is not free to ignore the $\tau(x)$ dependence of the couplings or the background vector field in the anomaly equation. But on the cycle the couplings are covariantly constant. Hence, the terms that vanish at fixed points because of the constancy of couplings also vanish for cycles, but now because they are covariantly constant. Finally, the validity of the limits in (5.3) and (5.4) needs to be established anew for limit cycles. However, the same method of conformal perturbation theory may be applied to establish the result. Since it is only scaling that is used in this step of the argument by LPR, the proof goes through as presented there.

\section{$6 \quad$ Summary and concluding remarks}

We have shown that the Komargodski-Schwimmer proof of the weak version of the $c$ theorem includes the more general case that a renormalization group flow goes from a fixed point or cycle to another fixed point or cycle. Regarding the strong version of the $c$-theorem, proven in perturbation theory by Jack and Osborn, we pointed out that the quantity that plays the role of $c$ is $\widetilde{B}_{b}$ (defined in (3.19)) which is closely related to the $a$-anomaly ( $\beta_{b}$ in the notation of Jack and Osborn); these quantities agree at fixed points and on cycles, but are not generally the same.

We presented a calculation of the Lie-algebra function of coupling constants $S$ introduced by Jack and Osborn. This is the first calculation of $S$ to an order (third) in the loop expansion where it does not vanish. We then proved that $S=0$ on fixed points and that $S$ precisely corresponds to the generator $Q$ of limit cycles when evaluated at any point on the limit cycle. This gives a major improvement on the method of searching for limit cycles: one merely needs to find zeroes of the beta functions to the first order in the loop expansion (second order for Yang-Mills couplings) and evaluate $S$ there. If $S=0$ the zero corresponds to a fixed point, while if $S=Q \neq 0$ the zero corresponds to a limit cycle with $Q$ the generator of the cycle.

We used these results to show that the trace of the stress-energy tensor vanishes on cycles, and hence that scale implies conformal invariance (perturbatively in unitary relativistic $d=4 \mathrm{QFT}$ ). If "theory space" is understood as the space of couplings of a model modulo the action of $G_{F}$ on these couplings (with $G_{F}$ the group of symmetries of the free Lagrangian), then cycles and fixed points are mapped to single points. It is remarkable that all such points describe in fact CFTs. 
Some questions remain which we intend to turn to in the future. Among them are:

- Are there renormalization group flows between fixed points and cycles?

- Are there limit cycles in four dimensions with bounded tree-level scalar potential?

- Are there any properties of cyclic CFTs that generically distinguish them from fixed point CFTs? In particular, does the current associated with the generator $S$ play a special role?

- Can a non-perturbative proof of the strong version of the $c$-theorem be given by extending the perturbative proof, say, by showing positivity of the metric $\chi_{I J}^{g}$ using dispersion relations?

- Do relativistic, unitary QFTs admit recursive RG flows that do not correspond to motions by generators in $G_{F}$ ?

We look forward to addressing these questions.

\section{Acknowledgments}

We are happy to acknowledge discussions with Hugh Osborn, Markus Luty, Joseph Polchinski and Riccardo Rattazzi that challenged our previous conclusion that theories on RG limit cycles are only scale-invariant. We thank David Pirtskhalava for numerous discussions and for his comments on the manuscript, as well as Ken Intriligator for discussions at the initiation of this project. This material is based upon work supported by the US Department of Energy under contract DOE-FG03-97ER40546 and in part by the US National Science Foundation under Grant No. 1066293 and the hospitality of the Aspen Center for Physics.

\section{A Ambiguities in RG functions}

It is well-known that anomalous-dimension matrices and beta functions are dependent on the renormalization scheme. Nevertheless, physical quantities obtained from the anomalous-dimension matrices and the beta functions which are relevant to the study of scale-invariant theories are, as expected, independent of the scheme [6].

It is however usually not appreciated that anomalous-dimension matrices and beta functions exhibit another freedom, mentioned briefly in the beginning of section 3, which we review here. For simplicity consider a theory of real multi-component scalars with bare Lagrangian

$$
\mathscr{L}_{0}=\frac{1}{2} \partial_{\mu} \phi_{0 a} \partial^{\mu} \phi_{0 a}-\frac{1}{4 !} g_{a b c d}^{0} \phi_{0 a} \phi_{0 b} \phi_{0 c} \phi_{0 d}
$$

There is an ambiguity in the definition of the wavefunction renormalization matrix $Z^{1 / 2}$, corresponding to the freedom of choosing $\tilde{Z}^{1 / 2}=O Z^{1 / 2}$ where $O^{T} O=\mathbb{1}$ [8]. In this appendix we study the effect of this ambiguity in the definition of RG functions. For simplicity we present this analysis in the flat background limit. Dimensional regularization is used throughout. 
Bare couplings and fields are related to the corresponding renormalized quantities by

$$
g_{I}^{0}=\mu^{k_{I} \epsilon}\left(g_{I}+L_{I}(g)\right), \quad \phi_{0}=\mu^{\delta \epsilon} \check{Z}(g) \phi,
$$

where $\check{Z}=Z^{1 / 2}$, and $\check{Z}-1$ and $L_{I}$ have expansions in $\epsilon$-poles starting at $1 / \epsilon$. The anomalous-dimension matrices and the beta functions, as well as the antisymmetric matrix $S$ of (3.11), are given by

$$
\hat{\gamma}=\delta \epsilon-k_{I} g_{I} \partial_{I} \check{Z}^{1}, \quad \hat{\beta}_{I}=-k_{I} g_{I} \epsilon-k_{I} L_{I}^{1}+k_{J} g_{J} \partial_{J} L_{I}^{1}, \quad S=-k_{I} g_{I} N_{I}^{1},
$$

where the superscript denotes residues of simple poles. The index carried by $k$ is exempt from the summation convention. In the present example $k_{I}=1$, but we keep it for generality. Since we are interested in ambiguities that arise because of different choices in the subtraction of infinite quantities, we assume that $O$ has an expansion in $\epsilon$-poles, $O=1+O^{1} / \epsilon+\cdots$, where $O^{1}$ is antisymmetric as required by $O^{T} O=\mathbb{1}$. Then, under the freedom mentioned above, it is easy to verify that the relevant quantities change as

$$
\check{Z}^{1} \rightarrow \check{Z}^{1}+O^{1}, \quad L_{I}^{1} \rightarrow L_{I}^{1}+\left(O^{1} g\right)_{I}, \quad N_{I}^{1} \rightarrow N_{I}^{1}-\partial_{I} O^{1},
$$

This induces a change in the anomalous-dimension matrix, the beta functions, and the antisymmetric matrix $S$ :

$$
\hat{\gamma} \rightarrow \hat{\gamma}-\omega, \quad \hat{\beta}_{I} \rightarrow \hat{\beta}_{I}+(\omega g)_{I}, \quad S \rightarrow S+\omega,
$$

where $\omega=k_{I} g_{I} \partial_{I} O^{1}$. This ambiguity, or "gauge" freedom, in the definition of anomalous dimensions and beta functions is usually resolved by requiring that the anomalousdimension matrix be symmetric. Note, however, that the trace of the stress-energy tensor, being a physical quantity, has to be invariant under this unphysical freedom. Indeed, this is obviously the case in (3.12). As we see $\hat{\beta}, \hat{\gamma}$ and $S$ are gauge-covariant, but $\widehat{B}_{I}=\hat{\beta}_{I}-(S g)_{I}$ and $\widehat{\Gamma}=\hat{\gamma}+S$ are gauge-invariant. Although RG flows are specified by $\hat{\beta}$, there is a gauge, defined by $\omega=-S$ so that $S=0$, in which $\widehat{B}=\hat{\beta}$ and $\widehat{\Gamma}=\hat{\gamma}$.

Finally, it is worth pointing out that $\widehat{B}_{I}$ could be seen as the proper vector field whose RG flows one should consider, and whose fixed points describe CFTs. This vector field does not admit cycles in perturbation theory.

\section{B The relation between the metrics $\chi_{i j}^{a}$ and $\chi_{i j}^{g}$}

The coefficient $c_{g}$ of LPR appears to play the role of the "metric" $\chi_{i j}^{g}$ in the consistency condition (2.13). As we mention in the end of section 2.2 and elaborate on further here, this is not the case. To see the connection with the work of JO, following LPR we write

$$
\Delta W_{\text {anomaly }}=\frac{1}{2} \int d^{4} x d^{4} y \tau(x) \tau(y)\langle\Theta(x) \Theta(y)\rangle,
$$

where $\Theta=\beta^{i} \mathcal{O}_{i}$, and therefore

$$
\frac{d}{d t} \Delta W_{\text {anomaly }}=\frac{1}{2} \int d^{4} x d^{4} y \tau(x) \tau(y) \frac{d}{d t}\langle\Theta(x) \Theta(y)\rangle .
$$


In ref. [14, Eq. (3.18b)] Osborn finds the RGE for the product of two local renormalized operators,

$$
-\frac{d}{d t}\left\langle\mathcal{O}_{i}(x) \mathcal{O}_{j}(0)\right\rangle+\partial_{i} \beta^{j}\left\langle\mathcal{O}_{i}(x) \mathcal{O}_{j}(0)\right\rangle+\partial_{j} \beta^{k}\left\langle\mathcal{O}_{i}(x) \mathcal{O}_{j}(0)\right\rangle=-\chi_{i j}^{a} \partial^{2} \partial^{2} \delta^{(4)}(x) .
$$

The quantity $\chi_{i j}^{a}$ can be thought of as the beta function associated with the counterterm needed in order to renormalize the correlator $\left\langle\mathcal{O}_{i}(x) \mathcal{O}_{j}(0)\right\rangle$. Now since $-d \beta^{i} / d t=\beta^{j} \partial_{j} \beta^{i}$, it is easy to see that

$$
\frac{d}{d t}\langle\Theta(x) \Theta(0)\rangle=\chi_{i j}^{a} \beta^{i} \beta^{j} \partial^{2} \partial^{2} \delta^{(4)}(x) .
$$

Using this in (B.1) we see that the metric of LPR is $-2 \chi^{a}$, which is always positive. This suggests the question "is there a relation between $\chi^{g}$ and $\chi^{a}$ ?"

In the specific example of a gauge theory with a simple gauge group $G$ and charged Dirac fermions in some representation, JO give [8, Eqs. (5.12)], at two loops,

$$
\chi^{a(2)}=-\frac{1}{2} \chi^{g(2)}=-\frac{n_{V}}{8 \pi^{2} g^{2}}\left[1+\left(17 C-\frac{20}{3} R\right) h\right], \quad h \equiv \frac{g^{2}}{16 \pi^{2}},
$$

where $\operatorname{tr}\left(t_{\text {adj }}^{a} t_{\text {adj }}^{b}\right)=C \delta^{a b}, R$ is similarly defined for the representation of the Dirac fermions, and $n_{V}=\operatorname{dim}(\mathrm{Adj})$ is the number of vectors. However, the relation $\chi^{g}=-2 \chi^{a}$ of (B.3) does not hold in general, and so the task of computing $\chi^{g}$ is complicated. Nevertheless, Weyl consistency conditions give the general relation between $\chi^{a}$ and $\chi^{g}$ [8, Eq. (3.23)]:

$$
\chi_{i j}^{g}+2 \chi_{i j}^{a}-\bar{\chi}_{i j k}^{a} \beta^{k}=-\beta^{k} \partial_{k} V_{i j}-\partial_{i} \beta^{j} V_{k j}-\partial_{j} \beta^{k} V_{i k},
$$

where $\zeta V_{i j}=\bar{\chi}_{i j k}^{a} k^{k} g^{k}$ (no sum over the index $k$ ), and $\bar{\chi}_{i j k}^{a}=\partial_{k} \chi_{i j}^{a}-\frac{1}{2}\left(\chi_{i k j}^{b}+\chi_{j k i}^{b}\right.$ ), with $\chi_{i j k}^{b}$ necessary to regulate infinities in three-point functions, and $\zeta$ defined as an operator counting the number of loops, whose form can be read off from $\mathcal{O}(\epsilon)$ terms of the finiteness condition $(3.9 \mathrm{e})$ of $\mathrm{JO}$ :

$$
\zeta V_{i j}=\left(1+k^{k} g^{k} \partial_{k}\right) V_{i j}+2 k^{i} V_{i j} \quad \text { (no sum over the index carried by } k \text { ) }
$$

(cf. JO's (3.16b)).

In our gauge-theory example (B.4) becomes

$$
\chi^{g}+2 \chi^{a}-\bar{\chi}^{a} \beta^{g}=-\beta^{g} \frac{\partial V}{\partial g}-2 \frac{\partial \beta^{g}}{\partial g} V, \quad \zeta V=\frac{1}{2} \bar{\chi}^{a} g,
$$

where $\zeta V=\left(2+\frac{1}{2} g \partial / \partial g\right) V=(2+h \partial / \partial h) V$, the beta function for the gauge coupling is

$$
\frac{1}{g} \beta^{g}=-\beta_{0} h-\beta_{1} h^{2}+\mathcal{O}\left(h^{3}\right), \quad \beta_{0}=\frac{1}{3}(11 C-4 R), \quad \beta_{1}=\frac{2}{3} C(17 C-10 R),
$$

and $\bar{\chi}^{a}=\partial \chi^{a} / \partial g-\chi^{b}$, where $\chi^{b}$ is given at two loops by $\chi^{b(2)}=\frac{n_{V}}{4 \pi^{2} g^{3}}\left(1+4 \beta_{0} h\right)$. It follows that

$$
\bar{\chi}^{a}=-\frac{n_{V}}{\pi^{2} g^{3}}\left[\beta_{0} h+\mathcal{O}\left(h^{2}\right)\right] .
$$


Expanding $V=v_{0}+v_{1} h+\cdots$ gives $\zeta V=2 v_{0}+3 v_{1} h+\cdots=\frac{1}{2} g \bar{\chi}^{a}$, or

$$
V=-\frac{n_{V} \beta_{0}}{64 \pi^{4}}+\mathcal{O}(h)
$$

With these results (B.5) gives

$$
\chi^{g}+2 \chi^{a}=-\frac{n_{V} \beta_{0}^{2}}{32 \pi^{4}} h+\mathcal{O}\left(h^{2}\right),
$$

and, therefore, beyond two-loop order, $\chi^{g} \neq-2 \chi^{a}$.

To summarize, the results of LPR correspond to using JO's $-2 \chi_{i j}^{a}$ as a metric, which however is not in general equal to JO's metric $\chi_{i j}^{g}$. Indeed, $\chi_{i j}^{g}+2 \chi_{i j}^{a}$ fails to vanish beyond the first few orders in the loop expansion. The positivity of $\chi_{i j}^{g}$ may also fail nonperturbatively (for example, if its perturbative expression has finite radius of convergence).

\section{How to calculate $N_{I}$ and $S$}

The calculation of JO's $N_{I}$ proceeds order by order in perturbation theory. In this appendix we calculate contributions to $N_{I}$ in a quantum field theory with real scalars and Weyl spinors up to two loops, and we also perform a three-loop calculation of the part of $N_{I}$ that is needed in order to compute $S$.

As can be seen from (3.1), in order to calculate $N_{I}$ we need to compute self-energies of scalars but with coupling constants as spectator fields. Equivalently, the calculation can be done by considering scalar self-energy diagrams and letting momentum come in from external legs and go out through couplings. From these diagrams we can then pick up the contribution linear in the momentum of the field and linear in the momentum of the coupling. After we antisymmetrize, we have a contribution to $N_{I}$.

It is perhaps helpful to remind the reader here that in a theory with scalars and fermions the $I$ index can be either $(a b c d)$ or $(a \mid i j)$. Let us also remark that $S$ appears first at three loops in a theory with scalars and spinors. The reason is easily seen from (3.11): a diagram that contributes to $N$ will only contribute to $S$ if it is not symmetric under $a \leftrightarrow b$. As it turns out there are no such diagrams in scalar self-energies at one and two loops, but there are four such diagrams at three loops. Consequently, even if the theory contains gauge fields, diagrams with gauge fields will not contribute to $S$ at three loops, but certainly will do so at higher order. Therefore, even in a gauge theory we don't need to include gauge fields in our leading-order calculation of $S$.

\section{C.1 One loop}

At one loop the calculation proceeds with no subtleties since renormalization is trivial, i.e., there are no subdivergences to be subtracted. The two diagrams that contribute to $N_{I}$ and their corresponding counterterms are shown in figure 2. A straightforward calculation gives

$$
\left(N_{c \mid i j}\right)_{a b}=-\frac{1}{16 \pi^{2} \epsilon} \frac{1}{2}\left(y_{a \mid i j}^{*} \delta_{b c}-y_{b \mid i j}^{*} \delta_{a c}\right)+\text { finite, }
$$

and there is of course a complex conjugate $\left(N_{c \mid i j}^{*}\right)_{a b}$. 

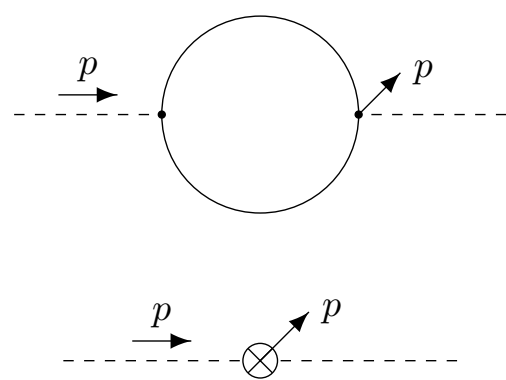
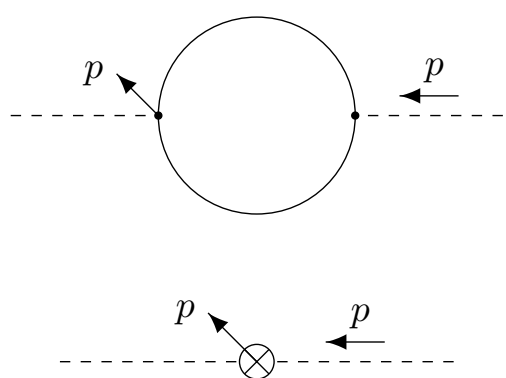

Figure 2. Diagrams that contribute to $N_{a \mid i j}$ at one loop and their corresponding counterterms.

In order to simplify the notation we write the result for the residue of the simple $\epsilon$-pole in $N_{I}$ in the form

$$
16 \pi^{2}\left(N_{I}^{1}\right)_{a b} \partial^{\mu} g_{I}=-\frac{1}{2}\left[\operatorname{tr}\left(y_{a} \partial^{\mu} y_{b}^{*}\right)+\text { h.c. }-\{a \leftrightarrow b\}\right],
$$

where $g_{I}$ on the left-hand side stands here for $y_{c \mid i j}$ or $y_{c \mid i j}^{*}$. Selecting the appropriate derivatives one easily reads off the corresponding $N_{I}^{1}$. Our result reproduces JO's equation (7.16) for $\rho_{I}$ when we use Dirac spinors.

\section{C.2 Two loops}

At two loops there are three Feynman diagrams that contribute to $N_{I}$, listed in figure 3 . The calculation of the residues of the simple $\epsilon$-poles of $N_{I}$ requires now a subtraction of subdivergences, something that proceeds, for the most part, in the usual way. However, there is a small subtlety, not seen in the usual treatments of renormalization, that we would like to point out. Clearly, the two right-most diagrams of figure 3 have subdivergences so we have to add to them the diagrams with the insertions of the corresponding counterterms. For the right-most diagram the graph with the insertion of the counterterm is

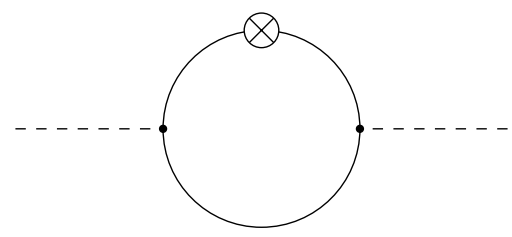

Now, when the momentum that comes in from, say the left external leg, flows out through the counterterm, then there are two diagrams that contribute, namely

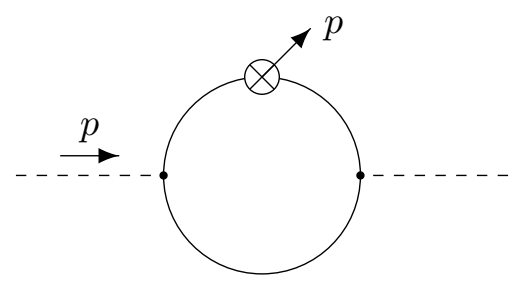

and

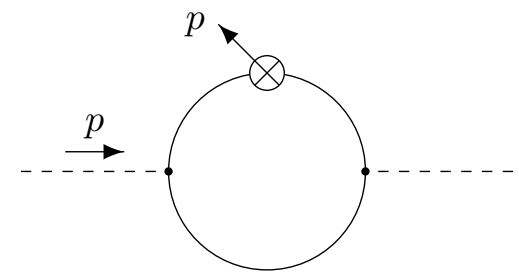



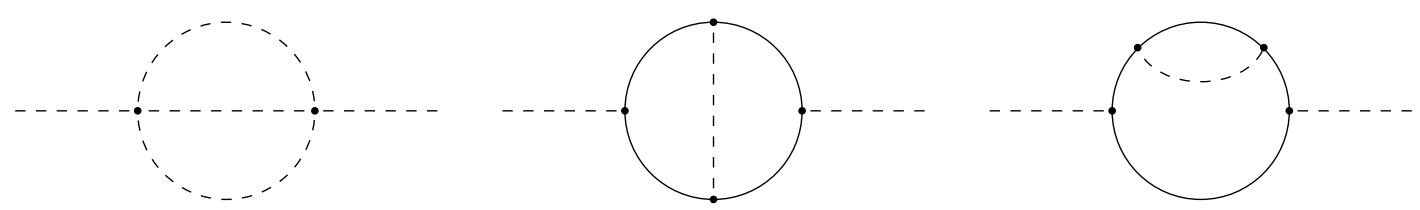

Figure 3. Feynman diagrams that contribute to $N_{I}$ at two loops.
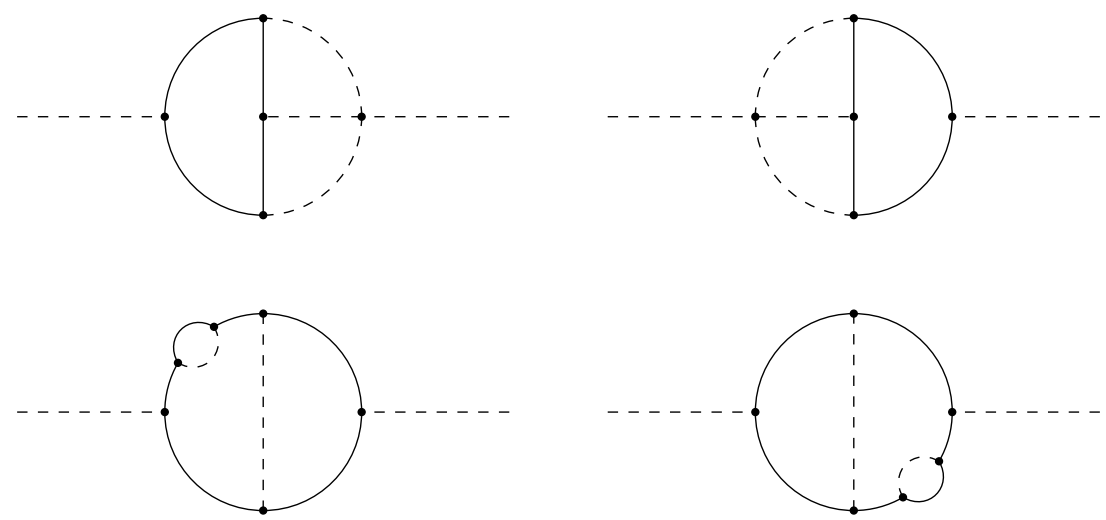

Figure 4. Three-loop diagrams that contribute to $N_{I}$ not symmetric under $a \leftrightarrow b$, and thus leading to contributions to $S$ at three loops.

where the momentum exits to the north-east or to the north-west depending on which vertex it flows out of in the original diagram in figure 3 . In both cases the counterterm is the same, but the diagram with the insertion of the counterterm is different as a result of the difference in the momentum of the internal leg that the counterterm picks up. That is, had we retained different momenta for the various vertices, there would be two momenta associated with the counterterm.

The two-loop result for $N_{I}^{1}$, previously unpublished, is

$$
\begin{aligned}
\left(16 \pi^{2}\right)^{2}\left(N_{I}^{1}\right)_{a b} \partial^{\mu} g_{I}= & -\frac{1}{24} \lambda_{a c d e} \partial^{\mu} \lambda_{b c d e}+\left[\frac{1}{4} \operatorname{tr}\left(y_{a}^{*} y_{c} \partial^{\mu} y_{b}^{*} y_{c}\right)\right. \\
& \left.+\frac{1}{8} \operatorname{tr}\left(y_{a}^{*} \partial^{\mu} y_{c} y_{c}^{*} y_{b}\right)+\frac{3}{8} \operatorname{tr}\left(y_{a}^{*} y_{c} y_{c}^{*} \partial^{\mu} y_{b}\right)+\text { h.c. }\right]-\{a \leftrightarrow b\}
\end{aligned}
$$

It follows that $S$ vanishes at this order. This can be seen, term by term (when antisymmetrized in $a$ and $b$ ) by replacing $g_{I}$ for $\partial^{\mu} g_{I}$.

\section{C.3 Three loops}

At three loops there are many diagrams that contribute to $N_{I}$, but only four are not symmetric under $a \leftrightarrow b$ and thus end up contributing to $S$. These diagrams are shown in figure 4 , and we here only compute their contributions to $N_{I}^{1}$. From these diagrams (and the corresponding counterterms), using the methods for the calculation of pole parts of 
three-loop diagrams given in [19], we find

$$
\begin{aligned}
\left(16 \pi^{2}\right)^{3}\left(N_{I}^{1}\right)_{a b} \partial^{\mu} g_{I} \supset & -\frac{1}{2} \operatorname{tr}\left(y_{a} \partial^{\mu} y_{c}^{*} y_{d} y_{e}^{*}\right) \lambda_{b c d e}-\frac{1}{3} \operatorname{tr}\left(y_{a} y_{c}^{*} \partial^{\mu} y_{d} y_{e}^{*}\right) \lambda_{b c d e} \\
& -\frac{1}{2} \operatorname{tr}\left(y_{a} y_{c}^{*} y_{d} \partial^{\mu} y_{e}^{*}\right) \lambda_{b c d e}-\frac{5}{24} \operatorname{tr}\left(y_{a} y_{c}^{*} y_{d} y_{e}^{*}\right) \partial^{\mu} \lambda_{b c d e} \\
& -\frac{1}{24} \operatorname{tr}\left(y_{b} \partial^{\mu} y_{c}^{*} y_{d} y_{e}^{*}\right) \lambda_{a c d e}-\frac{5}{24} \operatorname{tr}\left(y_{b} y_{c}^{*} \partial^{\mu} y_{d} y_{e}^{*}\right) \lambda_{a c d e} \\
& -\frac{1}{24} \operatorname{tr}\left(y_{b} y_{c}^{*} y_{d} \partial^{\mu} y_{e}^{*}\right) \lambda_{a c d e}-\frac{5}{24} \operatorname{tr}\left(\partial^{\mu} y_{b} y_{c}^{*} y_{d} y_{e}^{*}\right) \lambda_{a c d e} \\
& -\frac{7}{32} \operatorname{tr}\left(y_{a} \partial^{\mu} y_{c}^{*} y_{d} y_{d}^{*} y_{b} y_{c}^{*}\right)-\frac{7}{96} \operatorname{tr}\left(y_{a} y_{c}^{*} \partial^{\mu} y_{d} y_{d}^{*} y_{b} y_{c}^{*}\right) \\
& -\frac{23}{96} \operatorname{tr}\left(y_{a} y_{c}^{*} y_{d} \partial^{\mu} y_{d}^{*} y_{b} y_{c}^{*}\right)-\frac{7}{96} \operatorname{tr}\left(y_{a} y_{c}^{*} y_{d} y_{d}^{*} \partial^{\mu} y_{b} y_{c}^{*}\right) \\
& -\frac{7}{32} \operatorname{tr}\left(y_{a} y_{c}^{*} y_{d} y_{d}^{*} y_{b} \partial^{\mu} y_{c}^{*}\right)+\frac{1}{16} \operatorname{tr}\left(y_{a} \partial^{\mu} y_{c}^{*} y_{c} y_{d}^{*} y_{b} y_{d}^{*}\right) \\
& -\frac{5}{48} \operatorname{tr}\left(y_{a} y_{c}^{*} \partial^{\mu} y_{c} y_{d}^{*} y_{b} y_{d}^{*}\right)-\frac{1}{48} \operatorname{tr}\left(y_{a} y_{c}^{*} y_{c} \partial^{\mu} y_{d}^{*} y_{b} y_{d}^{*}\right) \\
& -\frac{7}{96} \operatorname{tr}\left(y_{a} y_{c}^{*} y_{c} y_{d}^{*} \partial^{\mu} y_{b} y_{d}^{*}\right)+\frac{1}{16} \operatorname{tr}\left(y_{a} y_{c}^{*} y_{c} y_{d}^{*} y_{b} \partial^{\mu} y_{d}^{*}\right) \\
& + \text { h.c. }-\{a \leftrightarrow b\}
\end{aligned}
$$

and since

$$
S \equiv-k_{I} N_{I}^{1} g_{I}=-N_{a b c d}^{1} \lambda_{a b c d}-\left(\frac{1}{2} N_{a \mid i j}^{1} y_{a \mid i j}+\text { h.c. }\right)
$$

we finally obtain

$$
\left(16 \pi^{2}\right)^{3} S_{a b}=\frac{5}{8} \operatorname{tr}\left(y_{a} y_{c}^{*} y_{d} y_{e}^{*}\right) \lambda_{b c d e}+\frac{3}{8} \operatorname{tr}\left(y_{a} y_{c}^{*} y_{d} y_{d}^{*} y_{b} y_{c}^{*}\right)+\text { h.c. }-\{a \leftrightarrow b\} .
$$

As already remarked in the main body, evaluating this on points in coupling space where we have found fixed points and cycles in refs. [4, 6, 7], we find that $S$ vanishes at all fixed points and equals $Q$ on all cycles.

Open Access. This article is distributed under the terms of the Creative Commons Attribution License which permits any use, distribution and reproduction in any medium, provided the original author(s) and source are credited.

\section{References}

[1] Z. Komargodski and A. Schwimmer, On Renormalization Group Flows in Four Dimensions, JHEP 12 (2011) 099 [arXiv: 1107.3987] [INSPIRE].

[2] A. Cappelli, G. D'Appollonio, R. Guida and N. Magnoli, On the c theorem in more than two-dimensions, JHEP (2000) [hep-th/0009119] [INSPIRE].

[3] A. Cappelli, R. Guida and N. Magnoli, Exact consequences of the trace anomaly in four-dimensions, Nucl. Phys. B 618 (2001) 371 [hep-th/0103237] [INSPIRE].

[4] J.-F. Fortin, B. Grinstein and A. Stergiou, Scale without Conformal Invariance: An Example, Phys. Lett. B 704 (2011) 74 [arXiv:1106.2540] [INSPIRE]. 
[5] J.-F. Fortin, B. Grinstein and A. Stergiou, Cyclic unparticle physics, Phys. Lett. B 709 (2012) 408 [arXiv:1110.1634] [INSPIRE].

[6] J.-F. Fortin, B. Grinstein and A. Stergiou, Scale without Conformal Invariance at Three Loops, JHEP 08 (2012) 085 [arXiv: 1202.4757] [INSPIRE].

[7] J.-F. Fortin, B. Grinstein and A. Stergiou, Limit Cycles in Four Dimensions, JHEP 12 (2012) 112 [arXiv:1206.2921] [INSPIRE].

[8] I. Jack and H. Osborn, Analogs for the c theorem for four-dimensional renormalizable field theories, Nucl. Phys. B 343 (1990) 647 [INSPIRE].

[9] J. Polchinski, Scale and conformal invariance in quantum field theory, Nucl. Phys. B 303 (1988) 226 [INSPIRE].

[10] J.-F. Fortin, B. Grinstein and A. Stergiou, Scale without Conformal Invariance: Theoretical Foundations, JHEP 07 (2012) 025 [arXiv:1107.3840] [InSPIRE].

[11] M.A. Luty, J. Polchinski and R. Rattazzi, The a-theorem and the Asymptotics of $4 D$ Quantum Field Theory, arXiv:1204.5221 [INSPIRE].

[12] A. Zamolodchikov, Irreversibility of the Flux of the Renormalization Group in a $2 D$ Field Theory, JETP Lett. 43 (1986) 730 [inSPIRE].

[13] J.L. Cardy, Is There a c Theorem in Four-Dimensions?, Phys. Lett. B 215 (1988) 749 [INSPIRE].

[14] H. Osborn, Weyl consistency conditions and a local renormalization group equation for general renormalizable field theories, Nucl. Phys. B 363 (1991) 486 [INSPIRE].

[15] J. Wess and B. Zumino, Consequences of anomalous Ward identities, Phys. Lett. B 37 (1971) 95 [INSPIRE].

[16] Z. Komargodski, The Constraints of Conformal Symmetry on RG Flows, JHEP 07 (2012) 069 [arXiv: 1112.4538] [INSPIRE].

[17] J.-F. Fortin, B. Grinstein, C.W. Murphy and A. Stergiou, On Limit Cycles in Supersymmetric Theories, arXiv:1210.2718 [INSPIRE].

[18] B. Grinstein, K.A. Intriligator and I.Z. Rothstein, Comments on Unparticles, Phys. Lett. B 662 (2008) 367 [arXiv:0801.1140] [INSPIRE].

[19] K.G. Chetyrkin, M. Misiak and M. Münz, $\beta$-functions and anomalous dimensions up to three loops, Nucl. Phys. B 518 (1998) 473 [hep-ph/9711266] [INSPIRE]. 